\title{
MODELING THE PAN-SPECTRAL ENERGY DISTRIBUTION OF STARBURST GALAXIES. III. EMISSION LINE DIAGNOSTICS OF ENSEMBLES OF EVOLVING H II REGIONS
}

\author{
Michael A. Dopita, Jörg Fischera, and Ralph S. Sutherland \\ Research School of Astronomy and Astrophysics, The Australian National University, \\ Cotter Road, Weston Creek, ACT 2611, Australia; michael.dopita@anu.edu.au \\ LisA J. KEWLEY \\ University of Hawai'i at Manoa, Institute for Astronomy, 2680 Woodlawn Drive, Honolulu, HI 96822 \\ Claus Leitherer \\ Space Telescope Science Institute, 3700 San Martin Drive, Baltimore MD 21218 \\ Richard J. Tuffs and Cristina C. Popescu \\ Max-Planck-Institut für Kernphysik, Saupfercheckweg 1, D-69117 Heidelberg, Germany
}

Wil van BReugel

Institute of Geophysics and Planetary Physics, Lawrence Livermore National Laboratory, L-413 Livermore, CA 94550

AND

Brent A. Groves

Max-Planck-Institut für Astrophysik, Karl-Schwarzschild-Strasse 1, 85741, Garching, Germany

Received 2006 April 3; accepted 2006 August 2

\begin{abstract}
We build, as far as theory will permit, self-consistent model H II regions around central clusters of aging stars. These produce strong emission line diagnostics applicable to either individual $\mathrm{H}$ II regions in galaxies or to the integrated emission line spectra of disk or starburst galaxies. The models assume that the expansion and internal pressure of individual $\mathrm{H}$ II regions is driven by the net input of mechanical energy from the central cluster, be it through winds or supernova events. This eliminates the ionization parameter as a free variable, replacing it with a parameter that depends on the ratio of the cluster mass to the pressure in the surrounding interstellar medium. These models explain why $\mathrm{H}$ II regions with low abundances have high excitation and demonstrate that at least part of the warm ionized medium is the result of overlapping faint, old, large, and low-pressure $\mathrm{H}$ II regions. We present line ratios (at both optical and IR wavelengths) that provide reliable abundance diagnostics for both single $\mathrm{H}$ II regions or for integrated galaxy spectra, and we find a number that can be used to estimate the mean age of the cluster stars exciting individual $\mathrm{H}$ II regions.
\end{abstract}

Subject headings: galaxies: abundances — galaxies: formation — galaxies: general — galaxies: starburst — ISM: abundances - $\mathrm{H}$ il regions

Online material: machine-readable tables

\section{INTRODUCTION}

Much of what we have learned about the chemical evolution of the universe, or of individual galaxies throughout cosmic time has been gleaned from the study of the spatially unresolved emission line spectra of distant galaxies (Steidel et al. 1996; Kobulnicky et al. 1999; Kobulnicky \& Zaritsky 1999; Kewley \& Kobulnicky 2005). This emission line spectrum arises from the ensemble average of $\mathrm{H}$ II regions within the galaxy, characterized by a wide range of physical parameters.

For example, in disk galaxies, the chemical abundance of heavy elements falls continuously from center to edge. In like manner, both the pressure and density of the interstellar medium in the disk drops exponentially with radius. For a given input of mechanical energy by the central star cluster, this means that the outermost $\mathrm{H}$ II regions expand more rapidly and are both larger and have lower internal pressure at a given age. However, the properties of the central cluster of a given mass change systematically with chemical abundance. Massive stars with higher chemical abundances lose more mass prior to supernova explosion, have stronger stellar winds, evolve more rapidly, and have lower effective temperatures while they are on the main sequence.
Finally, the temporal evolution of individual $\mathrm{H}$ II regions is extremely important in determining the integrated emission line spectrum. Leitherer et al. (1992) demonstrated the importance of aging of the $\mathrm{OB}$ stars in lowering the number of ionizing photons as the most massive stars evolve away from the zero-age main sequence (ZAMS) to become supergiants. Later, these stars enjoy a brief resurgence in their ionizing photon and mechanical energy production as they become Wolf-Rayet stars.

With so many temporal and physical variables, it is perhaps not surprising that the nebular modeling community (ourselves included) have in the past been reduced to making severe, even gross, oversimplifications in attempts to model the integrated strong emission line spectra of galaxies. Indeed, frequently only a single line ratio has been used to determine the metallicity of extragalactic $\mathrm{H}$ II regions or even of whole galaxies. This is the famous $R_{23}$ ratio; ([O II] $\lambda \lambda 3727,3729+[\mathrm{O}$ III] $\lambda \lambda 4959,5007) / \mathrm{H} \beta$.

This ratio was first proposed by Pagel et al. (1979). The logic for its use is impeccable, since it uses the two strongest lines of the strongest coolant of $\mathrm{H}$ II regions, and it should therefore be sensitive to the total oxygen abundance. By using two stages of ionization, it accounts for the emission in the bulk of the ionized nebular volume. However, the calibration of this ratio in terms of 
the abundance has proved to be very difficult and many different and often contradictory calibrations of $R_{23}$ are available, including Pagel et al. (1979, 1980), Edmunds \& Pagel (1984), McCall et al. (1985), Dopita \& Evans (1986), Torres-Peimbert et al. (1989), Skillman et al. (1989), McGaugh (1991), Zaritsky et al. (1994), Pilyugin (2000), and Kewley et al. (2002).

The basic cause of this difficulty in calibrating the $R_{23}$ ratio is common to many ratios formed from the flux of optical forbidden line divided by the flux of a recombination line of hydrogen. Initially, as $Z$ is increased, the forbidden line increases in flux, and the line ratio increases. However, as the abundance increases further, the cooling by forbidden lines lowers the electron temperature making it more difficult to collisionally excite the optical forbidden lines. Eventually, there comes a point at which an increase in abundance is matched by a decrease in collisional excitation and the line ratio reaches its maximum. Any further increase in $Z$ then leads to a decrease in the relative strength of the forbidden line. The ratio $R_{23}$, like other optical diagnostics, is therefore a two-valued function of $Z$. Because infrared lines have lower thresholds for collisional excitation, they remain monatonic functions of $Z$ up to much higher metallicities. Recognizing this physics, the $S_{23}$ ratio; ([S II $\left.] \lambda \lambda 6717,6731+\left[\mathrm{S}_{\text {III }}\right] \lambda \lambda 9069,9532\right) /$ $\mathrm{H} \beta$, or even more complex ratios, have been used either in the place of or to supplement the $R_{23}$ ratio (Dennefeld \& Stasinska 1983; Kennicutt \& Garnett 1996; Oey et al. 2002).

The calibration problems of the $R_{23}$ ratio are made worse by the scatter, at fixed $R_{23}$ and within each given branch, both between models and observational data, and among different models. The observational material is affected by uncertainty in the reddening correction applying to the $[\mathrm{O}$ II $] \lambda \lambda 3727,3729$, since classical extinction laws are usually used, rather than the attenuation laws, which more correctly apply to extended objects (Fischera et al. 2003; Fischera \& Dopita 2005). This point will be discussed more fully below. As far as the theoretical models are concerned, scatter in the relationship is easily generated, since the stellar atmospheres used for single stars can show very wide differences in the number of ionizing photons produced per unit frequency (Morisset et al. 2004).

Moving beyond the use of single line ratios, a more sophisticated analysis is made possible by the understanding that the strong line emission spectrum of an individual $\mathrm{H}$ II region is controlled by three physical parameters (Dopita \& Evans 1986). These parameters are the effective temperature of the exciting stars, the chemical abundance set (or "metallicity," $Z$ ), and the ionization parameter, defined either as the ratio of the mean ionizing photon flux to the mean atom density, $q$ or in its dimensionless form as the ratio of mean photon density to mean atom density $\mathcal{U}=q / c$. Thus, for a given stellar input spectrum a $\mathcal{U}: Z$ grid of models should provide the ideal means of interpreting the observational data (Kewley et al. 2002).

This analysis leaves open the issue of the age distribution of the exciting stars. The metallicity and the age of the exciting stars determines the energy distribution of the EUV photons. In the modeling hitherto, this problem is usually treated in two limits; the instantaneous burst approximation (for which zero age is often, but not universally assumed) or the continuous star formation approximation in which stars are assumed to be born continually within the ionized region (see Kewley et al. 2001 and references therein). All this modeling effectively assumes a single $\mathrm{H}$ II region of a given metallicity and ionization parameter. While such an approach is acceptable in attempts to understand the emission line spectrum of individual $\mathrm{H}$ II regions, it is certainly inadequate in attempts to interpret the integrated spectrum of a whole galaxy, or even of a complex of many $\mathrm{H}$ II regions.
The advent of the Starburst99 version 5 (Leitherer et al. 1999) stellar spectral synthesis code finally allowed the construction of fully self-consistent models for $\mathrm{H}$ II regions. For a given stellar initial mass function (IMF), this code delivers not only the panspectral distribution of photons, including ionizing photons as a function of age, but also provides tables of the time-dependent mechanical energy input of the stars including OB-star stellar winds, red giant winds, Wolf-Rayet winds, and the energy input from supernova explosions.

In the first paper of this series (Dopita et al. 2005, hereafter Paper I), we computed the (one-dimensional) evolution of H II regions around clusters of a single mass. We demonstrated how the size-to-age relationship is essentially determined by the density (or pressure) in the surrounding interstellar medium (ISM). This in turn determines the dust "temperature" described by the wavelength of the peak of the far-IR dust re-emission feature.

In the second paper of this series, Dopita et al. (2006a, hereafter Paper II), we investigated the role of metallicity and of the cluster mass function in controlling the excitation of the populations of $\mathrm{H}$ in regions in galaxies, be they disk or starburst. We demonstrated that the ionization parameter of $\mathrm{H}$ II regions, previously treated as a free variable, is determined at any time by the instantaneous ratio of the ionizing photon flux to the mechanical energy flux of the central stars. This is because the $\mathrm{H}$ II region is evolving as a mass-loss bubble pressurized by the combined ram pressure of the stellar wind and supernova explosions, so $\mathrm{H}$ II region density and $\mathrm{H}$ II region radius are closely coupled. Although this conclusion was obtained using a simple one-dimensional spherical evolution model for the $\mathrm{H}$ II region, it should remain valid in more complex geometries, since wherever unionized or molecular inclusions exist, these will be pressure confined by the hot shocked stellar wind gas, and this gas pressure is coupled with the overall size of the $\mathrm{H}$ II region, which itself determines the dilution of the radiation field of the central stars.

Because the effective temperature of the exciting stars increases as metallicity is lowered, but the mechanical energy flux in the stellar winds decreases toward to lower metallicity, lowabundance $\mathrm{H}$ II regions are characterized by higher ionization parameters, a fact that had been frequently noted by observers, but which had not previously been explained in a satisfactory manner.

Using these insights, in this third paper of the series, we proceed to the next stage of sophistication in modeling - the construction of time-dependent photoionization models of individual $\mathrm{H}$ II regions, and the construction of ensemble averages of aging $\mathrm{H}$ II regions to provide galaxy-wide averaged spectra at a given metallicity. Such models applied to individual $\mathrm{H}$ II regions make it possible to estimate the ages of the exciting stars from the positions of the observations on theoretical $\mathrm{H}$ II region isochrones. Previously, this could not be done, since the ionization parameter and the effective temperature of the cluster (determined by the aging of its stars) can both produce similar effects on the emitted spectrum. This degeneracy is raised thanks to the self-consistent geometry that we have developed, which includes a dynamical evolution of the $\mathrm{H}$ il region consistent with the properties of the stellar wind generated by the central cluster.

In this paper, we apply these new techniques to produce line ratio diagnostics that enable the independent determination of both the stellar ages and metallicities of the exciting clusters for individual $\mathrm{H}$ II regions. These should prove useful in the analysis of abundance gradients in resolved disk galaxies. More importantly, we develop line ratio diagnostics for the determination of abundances in ensembles of $\mathrm{H}$ II regions in starburst and normal galaxies. This should greatly assist in analyses of the strong 
emission lines of the more distant and unresolved galaxies, observed either at optical wavelengths or in the IR.

\section{MODELS}

\subsection{Codes}

We have used the Starburst99 version 5 code in its latest (2005) version to compute the pan-spectral distribution of clusters of stars with a piecewise fit to a Miller-Scalo IMF (Miller \& Scalo 1979) between 0.1 and $120 M_{\odot}$. The parameters of this fit are the same as given in Miller \& Scalo (1979) and were also tabulated by us in Paper II. Either the use of a Miller-Scalo IMF or of a Salpeter IMF would not noticeably affect the line ratio diagnostics presented in this paper, since the slopes of the IMF are almost identical above $10 M_{\odot}$. However, the choice of the IMF has a much larger effect on the total mass of the cluster, since this is determined by the choice of the power law below $10 M_{\odot}$.

Stellar atmosphere models for stars with plane parallel atmospheres are based on the Kurucz (1992) models as compiled by Lejeune et al. (1997). The fully line-blanketed Wolf-Rayet atmosphere models of Hillier \& Miller (1998) and the non-LTE O-star atmospheres of Pauldrach et al. (2001) have been incorporated into Starburst99 version 5 as described in Smith et al. (2002). The flux distributions following from these atmospheres are coupled to the stellar evolution models by the Geneva group. We used the so-called "high-mass-loss" tracks, which provide the best match to the observed stellar inventory in the HertzsprungRussell diagram (Leitherer et al. 1999).

For the IMF we used piecewise power-law fits to the Miller \& Scalo (1979) mass function, although we note that their paper, Miller \& Scalo (1979) represented their IMF as a truncated lognormal distribution (with its maximum at zero). However, they also represented it as a three-segment broken power law, which is the form of the IMF that Starburst99 currently accepts. The logarithmic slopes of the piecewise power-law fits, $\Gamma$, and the mass range applicable to each segment were given in Table 1 of Paper II.

For a given cluster mass, we used the stellar wind and supernova power output, as tabulated by the Starburst 99 code version 5 , to solve for the radius of the $\mathrm{H}$ II regions as a function of time given the pressure or, equivalently, the mean density in the ISM. This requires a Runge-Kutta integration of the equation of motion of the swept-up shell, as was done and described in both Paper I and Paper II.

The pressure in the $\mathrm{H}$ II region is the same as in the shocked stellar wind gas. This is given by the classical Castor et al. (1975) theory,

$$
P(t)=\frac{7}{(3850 \pi)^{2 / 5}}\left(\frac{250}{308 \pi}\right)^{4 / 15}\left[\frac{L_{\mathrm{mech}}(t)}{\mu m_{\mathrm{H}} n}\right]^{2 / 3} \frac{\mu m_{\mathrm{H}} n_{0}}{r(t)^{4 / 3}},
$$

where $L_{\text {mech }}(t)$ is the instantaneous production of mechanical energy by the cluster stars, $r(t)$ is the radius, $n_{0}$ is the mean atom density in the surrounding ISM, $n$ is the mean atom density in the ionized gas, and $\delta(t)=n / n_{0}$ is the instantaneous ratio of the density interior to the bubble to the density in the surrounding ISM.

In Paper II, we showed that the ionization parameter at the contact discontinuity in the swept-up shell of ISM, which can be treated as the inner boundary of the $\mathrm{H}$ II, region depends largely on the instantaneous properties of the exciting cluster stars:

$$
q(t) \propto \delta(t)^{3 / 2} S_{*}(t) / L_{\mathrm{mech}}(t),
$$

TABLE 1

Adopted Solar Chemical Abundance Ratios,

\begin{tabular}{|c|c|c|}
\hline Element & $\log \left[N_{\mathrm{X}} / n_{\mathrm{H}}\right]$ & $\log (D)$ \\
\hline 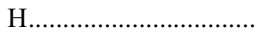 & 0.00 & 0.00 \\
\hline $\mathrm{He}$ & -1.01 & 0.00 \\
\hline С & -3.59 & -0.15 \\
\hline N & -4.22 & -0.23 \\
\hline О & -3.34 & -0.21 \\
\hline $\mathrm{Ne}$ & -3.91 & 0.00 \\
\hline $\mathrm{Mg}$ & -4.47 & -1.08 \\
\hline $\mathrm{Si}$ & -4.49 & -0.81 \\
\hline S & -4.79 & -0.08 \\
\hline $\mathrm{Ar}$ & -5.20 & 0.00 \\
\hline $\mathrm{Ca}$ & -5.64 & -2.52 \\
\hline $\mathrm{Fe}$ & -4.55 & -1.31 \\
\hline
\end{tabular}
$\log \left[n_{\mathrm{X}} / n_{\mathrm{H}}\right]$, ANd Depletion Factors $(D)$

where $S_{*}(t)$ is the instantaneous flux of ionizing photons from the central cluster.

The presence of the $\delta(t)$ factor in the above equation provides a weak coupling between the ionization parameter and both the pressure in the ISM, $P_{0}$, and the mass of the central cluster, $M_{\mathrm{cl}}$. Together, these determine the strength of the outer shock of the mass-loss bubble and therefore the compression factor through it. The appropriate scaling factors are $q \propto P_{0}^{-1 / 5}$ and $q \propto M_{\mathrm{cl}}^{1 / 5}$. Thus, once $P_{0}$ and $q \propto M_{\mathrm{cl}}^{1 / 5}$ are fixed, the ionization parameter is determined.

Using this dynamical model to derive the instantaneous size and ionization parameter of the $\mathrm{H}$ In region, the latest version of our code MAPPINGS IIIr (Sutherland \& Dopita 1993; Dopita et al. 2002; Groves et al. 2004) with the physical parameters defined in Paper I was used to compute photoionization models. The resulting model isobaric dusty $\mathrm{H}$ II regions were used to produce a table of emission lines, described in the Appendix. These enable us to compute the line ratio diagnostics described in this paper.

\subsection{Metallicities and Depletion Factors}

The solar abundance set adopted here is taken from Asplund et al. (2005). This incorporates the results of many papers that together have provided a self-consistent recalibration of the solar abundance. In many cases, these abundances are nearly a factor of 2 lower than those used previously (Anders \& Grevesse 1989). The corresponding logarithmic abundance ratios of the elements with respect to hydrogen are given in Table 1. For the purposes of the modeling, when we refer to solar metallicity, $Z_{\odot}$, we are using this set of abundances.

We note here that as a consequence of the rescaling of the solar abundances (which is not yet reflected in the stellar models), the Starburst99 "solar" abundance set is inconsistent with the new Asplund et al. (2005) abundance set. The Starburst99 code uses absolute metallicities of $Z=0.001,0.004,0.008,0.02$, and 0.04 , which makes the Starburst99 "solar" metallicity, $Z=0.02$, higher than the Asplund et al. (2005) "solar metallicity," $Z=0.016$. While this matters very little in terms of the number of ionizing photons emitted by the stars, it will mean that the computed stellar UV photon field will slightly softer than it should be, given the nebular abundance set.

The depletion factors from the gaseous phase determine the composition of the dust in the models. For these we have took the measured gas-phase abundances in the local interstellar cloud by Kimura et al. (2003) and used these to infer a set of local depletion factors consistent with the Asplund et al. (2005) abundance 


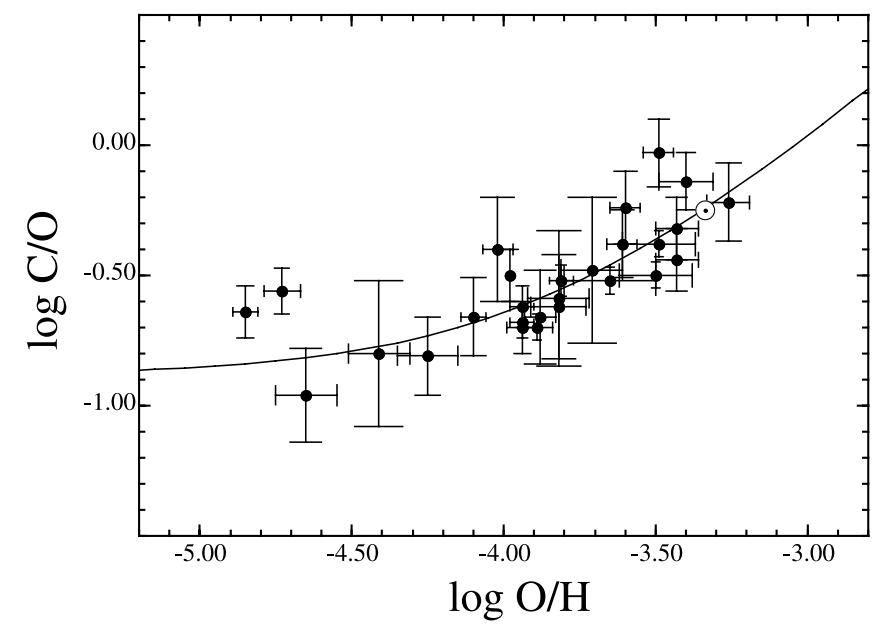

FIG. 1.-Fit given by eq. (5) is shown along with the HST observations of $\mathrm{H}$ II regions made by Garnett et al. (1999). The solar abundance from Asplund et al. (2005) is also marked with a Sun symbol $(\odot)$. The new solar abundance set eliminates the systematic difference between the solar and the $\mathrm{H}$ II region abundances, which was noted by Garnett et al.

set. The adopted depletion set is also given in Table 1. For models with nonsolar abundances, the depletion factors were held constant at solar values. This is equivalent to taking the dust-togas ratio to be proportional to metallicity.

Three elements do not scale simply with the metallicity. First, because of its high initial abundance as a result of nuclear burning in the big bang, helium scales only weakly with metallicity. For this element, we assume a primary nucleosynthesis component in addition to its primordial value. From Russell \& Dopita (1992) and Pagel et al. (1992) we infer the empirical relationship:

$$
\mathrm{He} / \mathrm{H}=0.0737+0.024 Z / Z_{\odot} .
$$

For both nitrogen and carbon there is clear evidence that these are both a primary nucleosynthetic element, dominant at low metallicity, and a secondary nucleosynthetic element once higher abundances are reached. Alternatively, both are produced in part by dredge-up in intermediate-mass stars, causing a delay in the onset of enrichment of these elements from this source. For nitrogen we have used a modified version of the form proposed by Groves et al. (2004):

$$
\mathrm{N} / \mathrm{H}=1.1 \times 10^{-5} Z / Z_{\odot}+4.9 \times 10^{-5} Z / Z_{\odot}^{2} .
$$

For carbon, we have used the HST data on the carbon abundances of $\mathrm{H}$ II regions as compiled by Garnett et al. (1999). This provides a fit of the same mathematical form as for $\mathrm{N}$ :

$$
\mathrm{C} / \mathrm{H}=6.0 \times 10^{-5} Z / Z_{\odot}+2.0 \times 10^{-4} Z / Z_{\odot}^{2} .
$$

The fit to the observations is shown in Figure 1.

\subsection{Parameters of the Photoionization Models}

Starburst99 version 5 allows us to study line ratios at five (fixed) absolute metallicities of $Z=0.001,0.004,0.008,0.02$, and 0.04 . For each of these abundance sets we have computed several families of $\mathrm{H}$ II region models with ages $0.2,0.5$, and 1.0 Myr and in further $0.5 \mathrm{Myr}$ steps up to a maximum age of 6.5 Myr. By this time, more than $96 \%$ of ionizing photons have been emitted, the $\mathrm{H}$ II regions have faded by factors of between 5 and 12 from their peak luminosity, and their surface brightnesses will have faded by much greater factors than these.

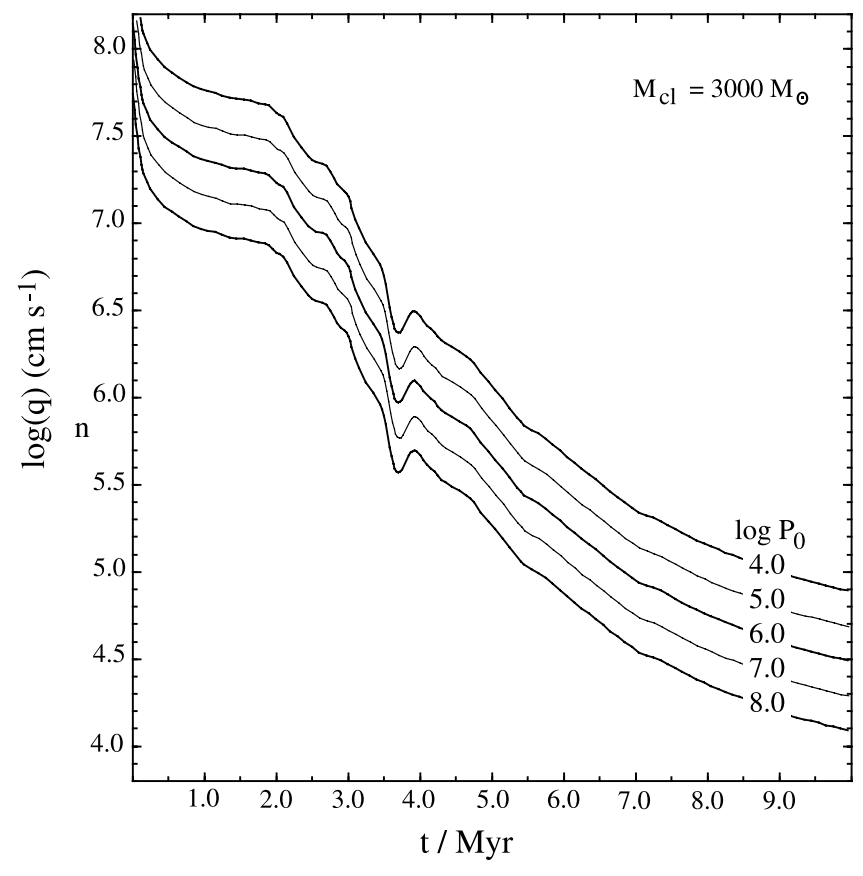

FIG. 2.-Computed run of ionization parameter at the inner edge of the $\mathrm{H}$ II region as a function of time and ISM pressure $\log P_{0} / k\left(\mathrm{~cm}^{-3} \mathrm{~K}\right)$ for a cluster having a mass of $3 \times 10^{3} M_{\odot}$ and assuming a Miller-Scalo IMF. A given choice of the variable $\mathcal{R}=\left(M_{\mathrm{cl}} / M_{\odot}\right) /\left(P_{0} / k\right)$ defines a unique run of the ionization parameter with time.

The ionization parameter is not a free variable in these models. The geometry of the gas with respect to the ionizing stars is instead determined by the equation of motion of the expanding $\mathrm{H}$ II region under the driving pressure of the stellar mass-loss and supernova explosions. This pressure also determines the density of the ionized plasma. As was shown in Paper II, the equation of motion and the pressure together lead us to infer that there is a weak coupling between the ionization parameter and both the pressure in the ISM, $P_{0}$, and the mass of the central cluster, $M_{\mathrm{cl}}$. Together, these determine the strength of the outer shock of the mass-loss bubble and therefore the compression factor through it. The appropriate scaling factors are $q \propto P_{0}^{-1 / 5}$ and $q \propto M_{\mathrm{cl}}^{1 / 5}$.

The ratio $\mathcal{R}=\left(M_{\mathrm{cl}} / M_{\odot}\right) /\left(P_{0} / k\right)$, with $P_{0} / k$, measured in cgs units $\left(\mathrm{cm}^{-3} \mathrm{~K}\right)$, uniquely determines the run of ionization parameter with time. It is this variable that replaces the ionization parameter and other geometrical considerations in our models. In Figure 2 we show the run of the computed ionization parameter $q$ as a function of time and ISM pressure for a solar abundance cluster with $M_{\mathrm{cl}}=3 \times 10^{3} M_{\odot}$ and having a Miller-Scalo IMF. The (dimensional) ionization parameter $q$ is related to the more frequently used (dimensionless) ionization parameter $\mathcal{U}$ by $\mathcal{U}=q / c$.

The run of $q$ with time is computed using the Starburst 99 output, and solving for the time dependent radius and internal pressure of the mass-loss bubble as described above. In the early phases $(t<2 \mathrm{Myr})$ the ratio of ionizing photon flux to mechanical energy flux is almost constant, and the changes in $q$ mostly reflect the size evolution of the mass-loss bubble. After $2 \mathrm{Myr}$, the ionizing photon flux rapidly decreases as massive stars evolve to red supergiants, and then the mechanical energy flux increases as a result of Wolf-Rayet stellar winds. Both of these lead to a strong decline in $q$. At around 3.5 Myr, supernova explosions add to the internal pressure of the bubble, sharply decreasing the $q$ in the ionized gas and reaccelerating the expansion of the mass-loss bubble. At later times, the progressive death of the high-mass stars leads to further declines in the ionizing photon flux, but the decline 

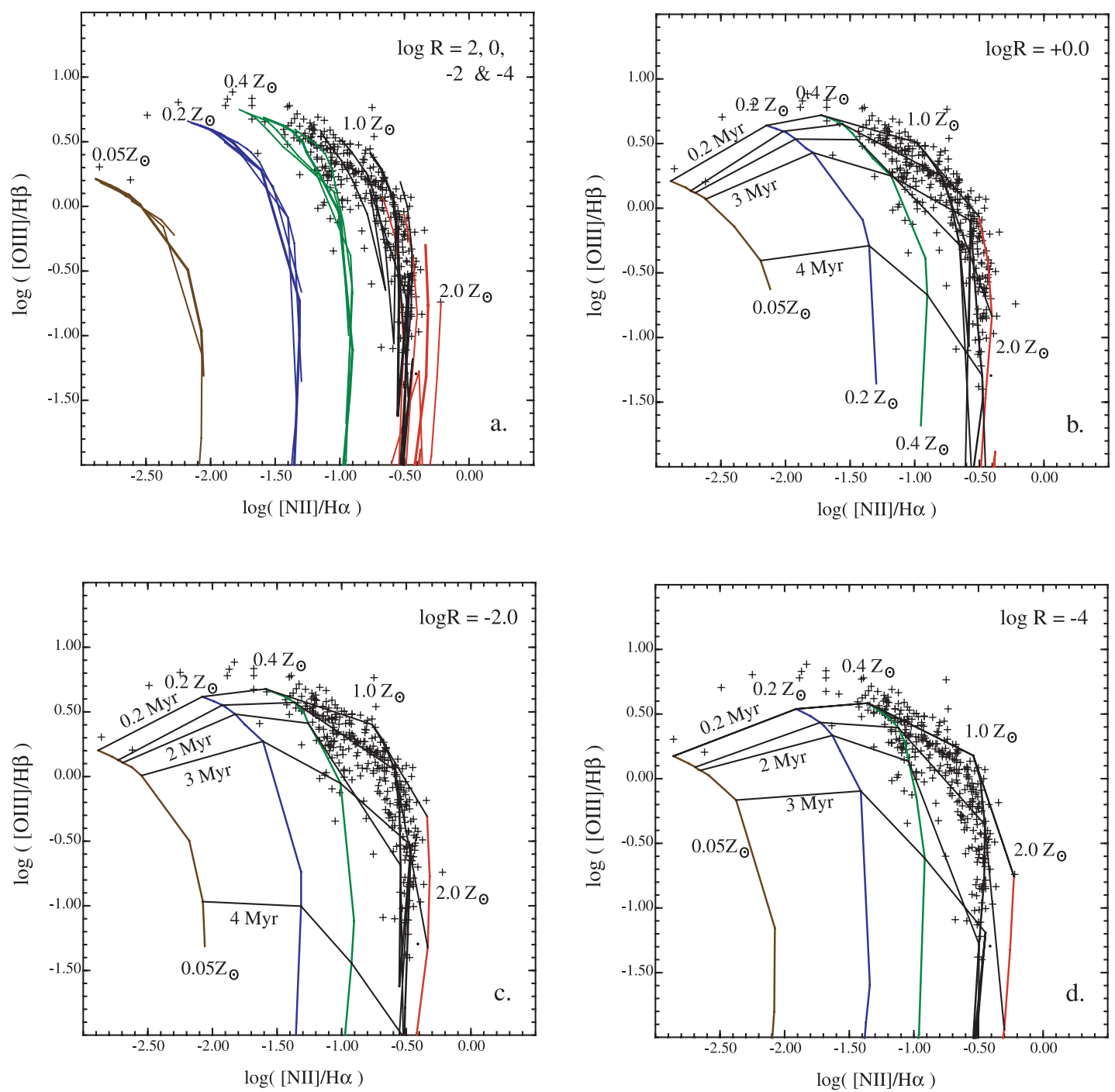

FIG. 3. - The [ $\mathrm{N}$ II] $\lambda 6584 / \mathrm{H} \alpha$ ratio vs. the $\left[\mathrm{O}\right.$ III] $\lambda 5007 / \mathrm{H} \beta$ ratio. The abundances are coded by color: for $Z / Z_{\odot} 0.05,0.2,0.4,1.0$, and 2.0 the colors are brown, blue, green, black, and red, respectively. In $(a)$ we plot all models of a given metallicity, and in the remaining panels we plot aging tracks at each metallicity and the corresponding isochrones for three likely values of the $R$ parameter: $\log \mathcal{R}=0,-2$, and -4 , respectively.

in $q$ is slower because the internal pressure of the mass-loss bubble also declines, partially offsetting the decline in ionizing photon flux.

The theoretical ionization parameter rather strongly dependent on the chemical abundance. It is driven by two factors. First, at higher abundance, the stellar wind has a higher opacity and therefore absorbs a greater fraction of the ionizing photons, reducing the $q$ in the surrounding $\mathrm{H}$ II region. Second, the atmosphere scatters the photons emitted from the photosphere more efficiently when the atmospheric abundances are higher, leading to a greater conversion efficiency from luminous energy flux to mechanical energy flux in the stellar wind base region. This also leads to a diminution of $q$ in the surrounding $\mathrm{H}$ II region. These factors acting together provide a sensitivity to metallicity of $q \propto Z^{-0.8}$, approximately.

As described above at any age $t$, the instantaneous ionization parameter - which determines the excitation of the $\mathrm{H}$ II regionis governed by the ratio of the mass of the cluster and the pressure in the surrounding ISM. Since cluster masses may vary between $\sim 100$ and $10^{6} M_{\odot}$, and the likely range of ISM pressures are between $10^{4} \mathrm{~cm}^{-3} \mathrm{~K}<P_{0} / k<10^{7} \mathrm{~cm}^{-3} \mathrm{~K}$, it follows that reasonable values for $\mathcal{R}$ fall within the range $-6<\log \mathcal{R}<+2$. We have therefore computed, for each metallicity, an age se- quence of model $\mathrm{H}$ II regions having $\log \mathcal{R}=-6,-4,-2,0$, and +2 . For a given metallicity, the instantaneous value of the ionization parameter depends on $\mathcal{R}^{1 / 5}$, as we have shown in Paper II. Note that a particular choice of $\mathcal{R}$ is not tied to any particular cluster mass. Thus, a cluster of $10^{5} M_{\odot}$ would be characterized by $\log \mathcal{R} \sim 0.6$ in the solar vicinity, and $\log \mathcal{R} \sim-1.0$ in a highpressure starburst environment.

It is worth noting at this point that this parameterization of the problem of evolving $\mathrm{H}$ II regions only remains valid for as long as stochastic variation in the cluster membership may be ignored. This means that the exciting cluster has to be massive enough that the upper IMF is adequately populated with stars so as to avoid stochastic variations in either the mass-to-light ratio or the effective temperature of the cluster stars. This effectively limits the cluster mass for which these models are valid, $M_{\mathrm{cl}} \geq 10^{3} M_{\odot}$.

Together, the two parameters $Z / Z_{\odot}$ and $\log \mathcal{R}$ define a unique $\mathrm{H}$ II region spectrum, with one caveat, namely, that if the density of the ionized gas becomes too high, then collisional de-excitations of forbidden lines will alter the emergent spectrum. This only becomes a concern at the very highest values of ISM pressure either within ultracompact $\mathrm{H}$ II regions (as computed by Dopita et al. 2006a) or else in starburst environments with $P_{0} / k>10^{7} \mathrm{~cm}^{-3} \mathrm{~K}$. 

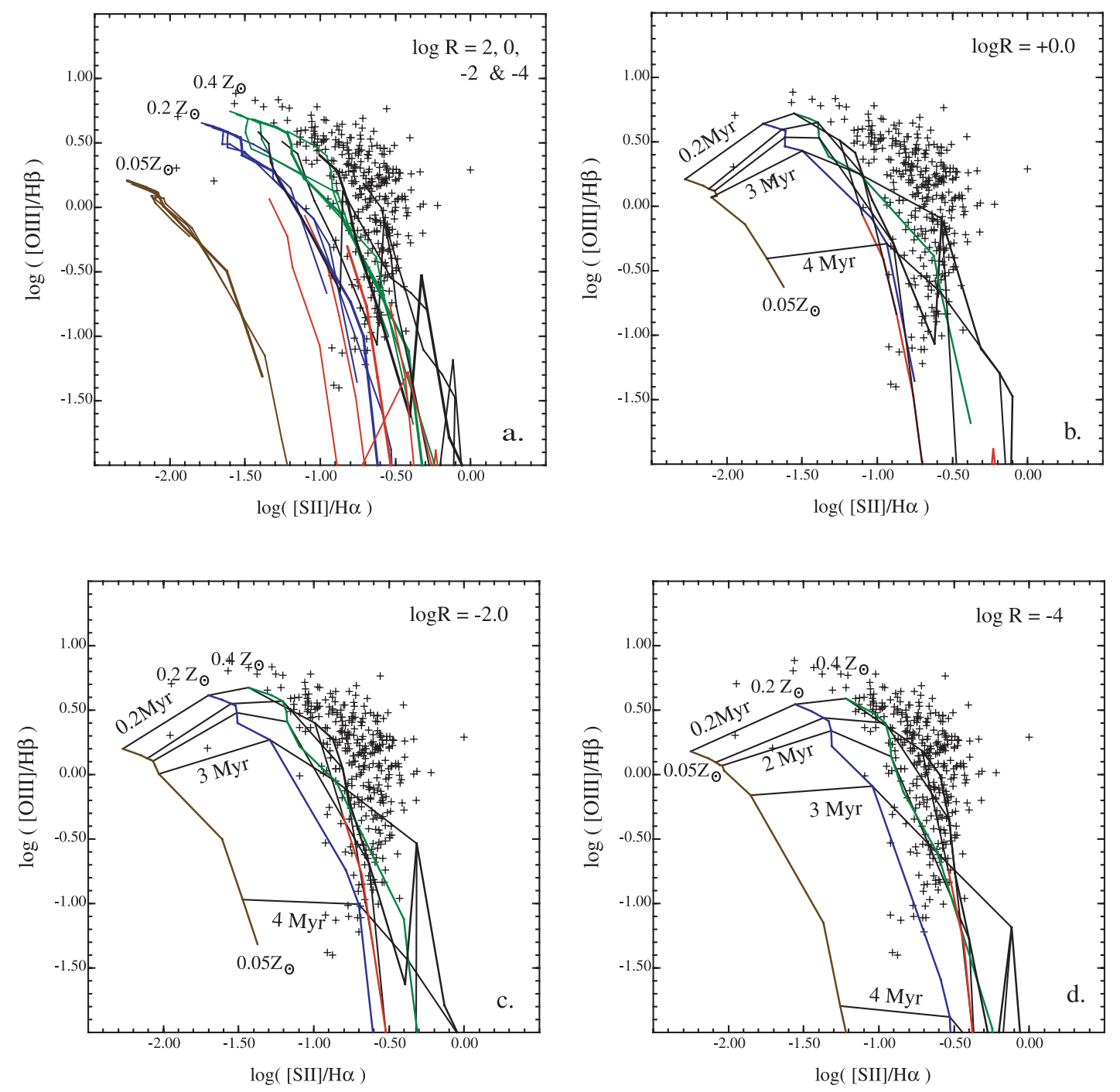

Fig. 4. - Same as Fig. 3 but for the second of the V\&O diagnostics, the $[\mathrm{S} \mathrm{II]} \lambda \lambda 6717,6731 / \mathrm{H} \alpha$ ratio vs. the $[\mathrm{O}$ III] $] / \mathrm{H} \beta$ ratio.

For the current purposes, we have computed the particular case of $P_{0} / k=10^{6} \mathrm{~cm}^{-3} \mathrm{~K}$, which constrains the electron density in the $\mathrm{H}$ II region below $\sim 100 \mathrm{~cm}^{-3}$, except at the very earliest times and in the models of highest metallicities, where it may reach $\sim 10^{3} \mathrm{~cm}^{-3}$.

For each model, we have prepared tabular data for all the strong lines between the Lyman limit and $88 \mu \mathrm{m}$, from which we form $\mathrm{H} \beta$ flux-weighted time-averaged spectra to represent the integrated spectrum for a given $Z / Z_{\odot}$ and $\log \mathcal{R}$. The set of strong spectral line intensities with respect to $\mathrm{H} \beta$ are available online. The form of the tables is given in the Appendix, Tables 2-7. The code also returns the intensities of very many more fainter lines, which are not listed here.

\section{H II REGION EMISSION LINE DIAGNOSTICS}

\subsection{Optical Lines}

\subsubsection{Veilleux \& Osterbrock Diagnostics}

The diagnostics that have been most frequently used in the classification of the nature of nebular excitation are those proposed by Veilleux \& Osterbrock (1987, hereafter V\&O). In these, the $\left[\mathrm{N}\right.$ II] $\lambda 6584 / \mathrm{H} \alpha$ the $\left[\mathrm{S}_{\text {III }} \lambda \lambda 6717,6731 / \mathrm{H} \alpha\right.$ or the $\left[\mathrm{O}_{\mathrm{I}}\right] \lambda 6300 /$ $\mathrm{H} \alpha$ are plotted against the $[\mathrm{O}$ III] $\lambda 5007 / \mathrm{H} \beta$ ratio.
These $\mathrm{V} \& \mathrm{O}$ ratios have the virtue of only using lines that are close together in wavelength in forming the ratio, so errors due to uncertain reddening corrections are avoided. These plots also nicely separate $\mathrm{H}$ II regions from regions excited by active galactic nuclei (Seyfert or LINER nuclei) and from shock-excited objects. The $\mathrm{H}$ II regions form an extremely tight sequence, particularly in the diagram using the $[\mathrm{N}$ II $] \lambda 6584 / \mathrm{H} \alpha$ ratio.

The results of our modeling for the $[\mathrm{N}$ II] $\lambda 6584 / \mathrm{H} \alpha$ versus the $[\mathrm{O}$ III $] / \mathrm{H} \beta$ ratio is shown in Figure 3. Because of the difficulty of representing so many theoretical models on the same figure, this is presented here in color, and in four panels. The first panel shows models of all ages for each metallicity, color coded for clarity. The model series corresponding to $\log \mathcal{R}=-2$ is drawn bold, since this is our best guess of the most likely value of $\log \mathcal{R}$ in disk galaxies $\left(10^{2} \leq M_{\mathrm{cl}} / M_{\odot} \leq 10^{4}\right.$ and $10^{4} \mathrm{~cm}^{-3} \mathrm{~K} \leq P / k \leq$ $\left.10^{6} \mathrm{~cm}^{-3} \mathrm{~K}\right)$. The remaining three panels show the complete grid of models for $\log \mathcal{R}=0,-2$, and -4 , respectively. Isochrones are plotted for $0.2,1.0,2.0,3.0$, and 4.0 Myr.

In this figure, the observational data sets for individual $\mathrm{H}$ II regions are presented as crosses. The data are drawn from van Zee et al. (1998), Kennicutt \& Garnett (1996), Dennefeld \& Stasinska (1983), Walsh \& Roy (1997), and Roy \& Walsh (1997), and the extension of the data set to low abundances is from Pagel et al. (1992). 

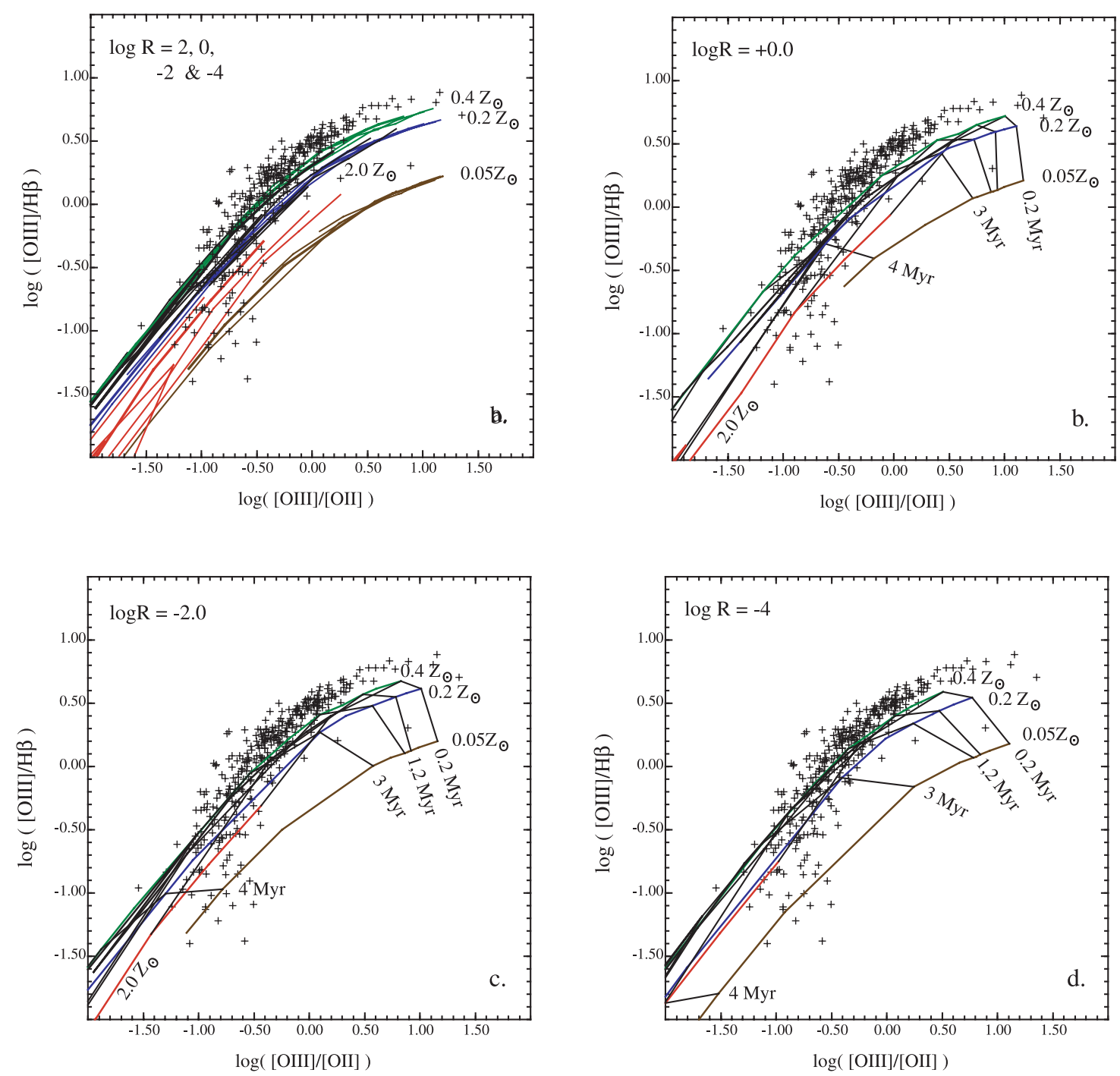

FIg. 5. - Same as Fig. 3 but for the $[\mathrm{O}$ III] $\lambda 5007 /[\mathrm{O}$ II] $\lambda \lambda 3727,3729$ vs. the $[\mathrm{O}$ III] $\lambda 5007 / \mathrm{H} \beta$ ratio. This is the diagnostic first used by BPT to separate AGN from $\mathrm{H}$ II regions. High-metallicity $\mathrm{H}$ II regions $\left(Z / Z_{\odot}>2\right)$ are located in the lower left-hand corner of this diagram. Note also how the low-metallicity models fall systematically below the main sequence of observed $\mathrm{H}$ II regions.

The reason why the observed $\mathrm{H}$ II regions on a $[\mathrm{N}$ II $] / \mathrm{H} \alpha$ versus the $[\mathrm{O}$ III $] / \mathrm{H} \beta$ form such a narrow sequence is quite evident. First, to be bright, and therefore selected for observation, observed $\mathrm{H}$ II regions should have an age less than $\sim 3$ Myr. In this age range, the isochrones of all models of different abundances fall into the very narrow strip defined by the observations. Although it depends somewhat on the assumed value of $\log \mathcal{R}$, it is clear that no observed $\mathrm{H}$ II region has an age greater than 3-4 Myr, consistent with the presumption of strong observational selection against old $\mathrm{H}$ il regions.

This figure should be compared with Figure 2 of Dopita et al. (2000) in which a grid of models in $Z / Z_{\odot}$ and $q$, the ionization parameter, were presented. It is clear that, in order to have a lowmetallicity $\mathrm{H}$ II region with a low ionization parameter, the $\mathrm{H}$ II region has to be faint and old and would therefore not have been observed in these surveys. For metallicities greater than about $Z / Z_{\odot} \sim 0.5$, the models are highly degenerate in terms of both the metallicity and the cluster age. This accounts for the very tight distribution of observed points in the region of the plot where $\log ([\mathrm{O} \mathrm{III}] / \mathrm{H} \beta)<0.0$.

Since the models of Dopita et al. (2000) were run, the EUV field predicted by the Starburst 99 version 5 code has softened, thanks to improvements in the treatment of extended atmospheres. This leads both to the reduction in the limiting $[\mathrm{N}$ II] $/ \mathrm{H} \alpha$ ratio seen in Figure 3, which improves the fit with the observations, and the decrease in the limiting $[\mathrm{O}$ III] $/ \mathrm{H} \beta$ ratio, which tends to make the fit somewhat worse at the low-metallicity end.

Note that the aging tracks make a sharp zig-zag causing the $3 \mathrm{Myr}$ isochrone to lie above the $2 \mathrm{Myr}$ isochrone for metallicities above solar. This is particularly visible in Figure $3 c$ and is the result of the appearance of the Wolf-Rayet stars at ages $\sim 3 \mathrm{Myr}$, which briefly harden the overall stellar radiation field before they themselves explode as Type II supernovae.

The fit between theory and observation is much poorer when we consider the second of the Veilleux \& Osterbock diagrams, which plots the [S II] $\lambda \lambda 6717,6731 / \mathrm{H} \alpha$ ratio versus the [O III] $\lambda 5007 / \mathrm{H} \beta$ ratio. This is shown in Figure 4. In general, the predicted [S II] lines are weaker than observed, by as much as a factor of 2. A similar effect was seen in our earlier modeling (see Fig. 3 of Dopita et al. 2000) and is not fully understood.

The $\left[\mathrm{S} \mathrm{II}_{\mathrm{II}} \lambda \lambda 6717,6731 / \mathrm{H} \alpha\right.$ ratio is more ambiguous than the [N II] $26584 / \mathrm{H} \alpha$ ratio if we wanted to use it as an abundance indicator, particularly at high metallicity. However, the $0.2-3 \mathrm{Myr}$ isochrones still define a narrow range in line ratio space, albeit 

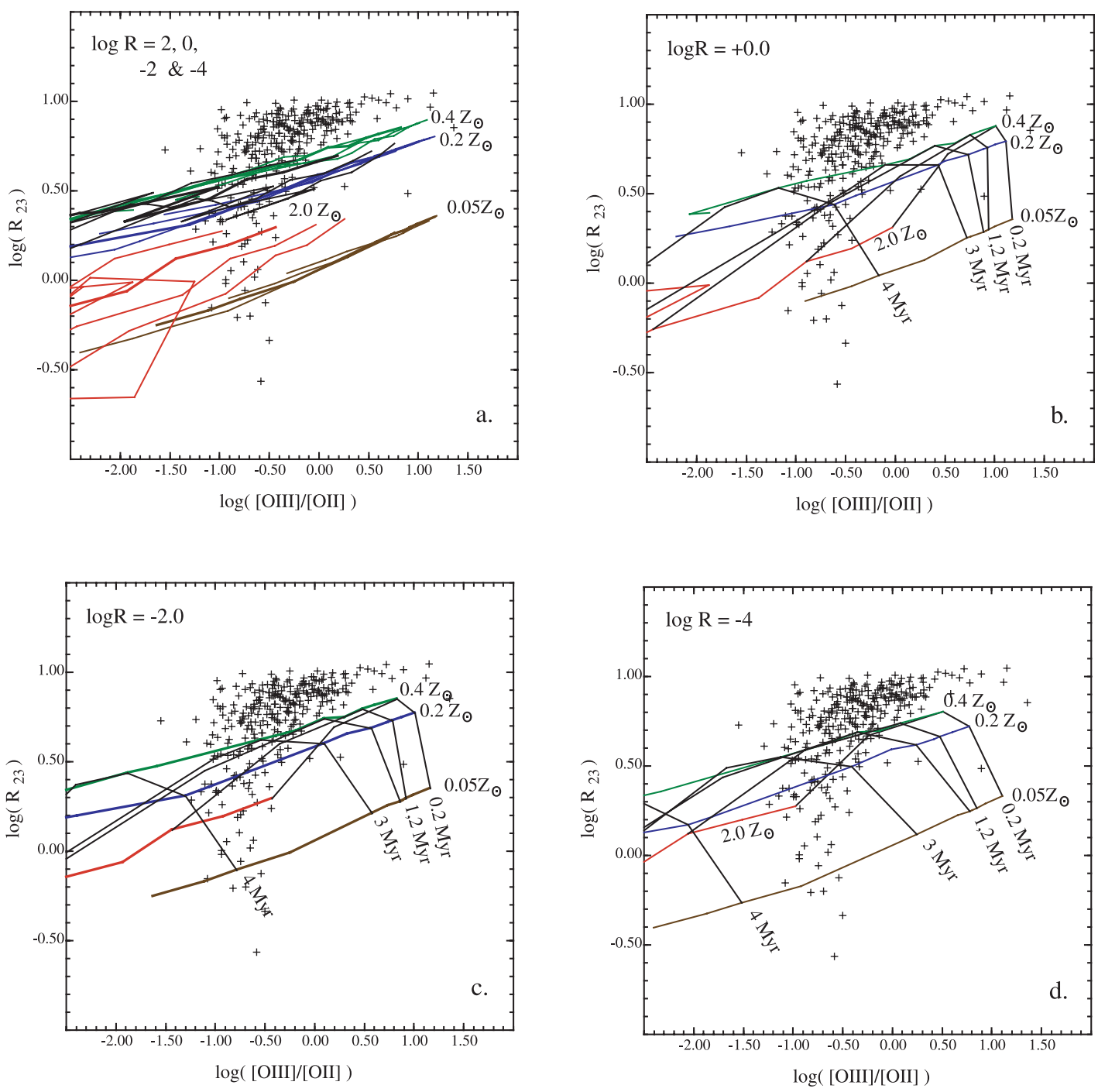

Fig. 6. - Same as Fig. 3 but for the $R_{23}$ ratio vs. the $[\mathrm{O}$ III $] 25007 /[\mathrm{O}$ I $] \lambda \lambda 3727,3729$ ratio. In $(b)-(d)$ the solar metallicity track has been omitted for clarity. This shows that the traditional $R_{23}$ ratio is not a good abundance diagnostic in the range $0.2 \leq Z / Z_{\odot} \leq 1.5$, approximately, and that it is quite sensitive to the nebular excitation. The theoretical tracks are, however, a rather poor fit to the observations. This is because the models have [O m] $\lambda \lambda 4959,5007$ and, even more so, $[\mathrm{O}$ II] $\lambda \lambda 3727,3729$, weaker than those observed.

not exactly coincident with the observations. The reasons for such a discrepancy between theory and observation in this as well as in other line ratio plots will be discussed in $\S 5$.

\subsubsection{The BPT Diagram}

Baldwin et al. (1981, hereafter BPT) first proposed the use of the $[\mathrm{O}$ III $] \lambda 5007 /\left[\mathrm{O}_{\text {III }}\right] \lambda \lambda 3727,3729$ ratio versus the $\left[\mathrm{O}_{\text {III }}\right] \lambda 5007 /$ $\mathrm{H} \beta$ line ratio as a diagnostic both to separate active nuclei from $\mathrm{H}$ II regions and also to provide an excitation sequence for $\mathrm{H}$ II regions. Our results for this line ratio are shown in Figure 5. The $[\mathrm{O} \mathrm{III}] /[\mathrm{O} \mathrm{II}]$ ratio is an excitation-sensitive ratio, sensitive to the ionization parameter in the nebula. Because of their higher ionization parameters and their central stars with higher effective temperatures at a given age, the low-metallicity $\mathrm{H}$ II regions are characterized by systematically higher $[\mathrm{O}$ III $] /[\mathrm{O}$ II] ratios. Like all other ratios of optical forbidden lines to recombination lines, for a given stellar age, the $[\mathrm{O}$ III] $\lambda 5007 / \mathrm{H} \beta$ ratio starts off low at low metallicity, reaches a maximum at a certain metallicity, and then declines swiftly at higher metallicities. The reason for this was explained in the introduction in the context of the $R_{23}$ ratio. As a consequence, the BPT diagram is highly degenerate in metallicity in the range $0.2 \leq Z / Z_{\odot} \leq 2$, explaining the tightness of the observed $\mathrm{H}$ II region sequence. This means, however, that the [O III]/
$\mathrm{H} \beta$ ratio is of little use as an abundance diagnostic except when $Z / Z_{\odot} \leq 0.2$. Even then it requires the observation of the $[\mathrm{O}$ III $] /$ [O II] ratio before an oxygen abundance can be derived from it.

Although the theoretical sequence has much the same form as defined by the observations, it is notable that, for all values of the $[\mathrm{O}$ III $] /[\mathrm{O}$ II $]$ ratio, the maximum of the $\left[\mathrm{O}_{\mathrm{III}}\right] / \mathrm{H} \beta$ lies below the maximum defined by the observations. Furthermore, even for the highest value of $\log \mathcal{R}$, the zero-age models do not reach to the maximum $\left[\mathrm{O}_{\mathrm{III}}\right] /[\mathrm{O} \mathrm{II}]$ ratio defined by the observations. As discussed in $\S 5$, below, both of these discrepancies can be ascribed to a single source, namely that the input spectrum we are using in the models is somewhat "softer" than the EUV spectrum found in real $\mathrm{H}$ II regions.

\subsubsection{The $R_{23}$ Ratio}

As described in the introduction, the $R_{23}$ ratio has had a long and checkered history as a metallicity indicator. However, the sensitivity of the $[\mathrm{O}$ III] $\lambda 5007 / \mathrm{H} \beta$ ratio to the cluster age shown in Figure 5 suggests that we should plot the $R_{23}$ ratio against the $[\mathrm{O}$ III] $/[\mathrm{O}$ II] ratio in order to be able to investigate the age sensitivity of the $R_{23}$ ratio. This diagnostic is shown in Figure 6.

For this diagnostic the fit between theory and observation is quite poor, due mainly to the fact that the $R_{23}$ ratio is systematically 

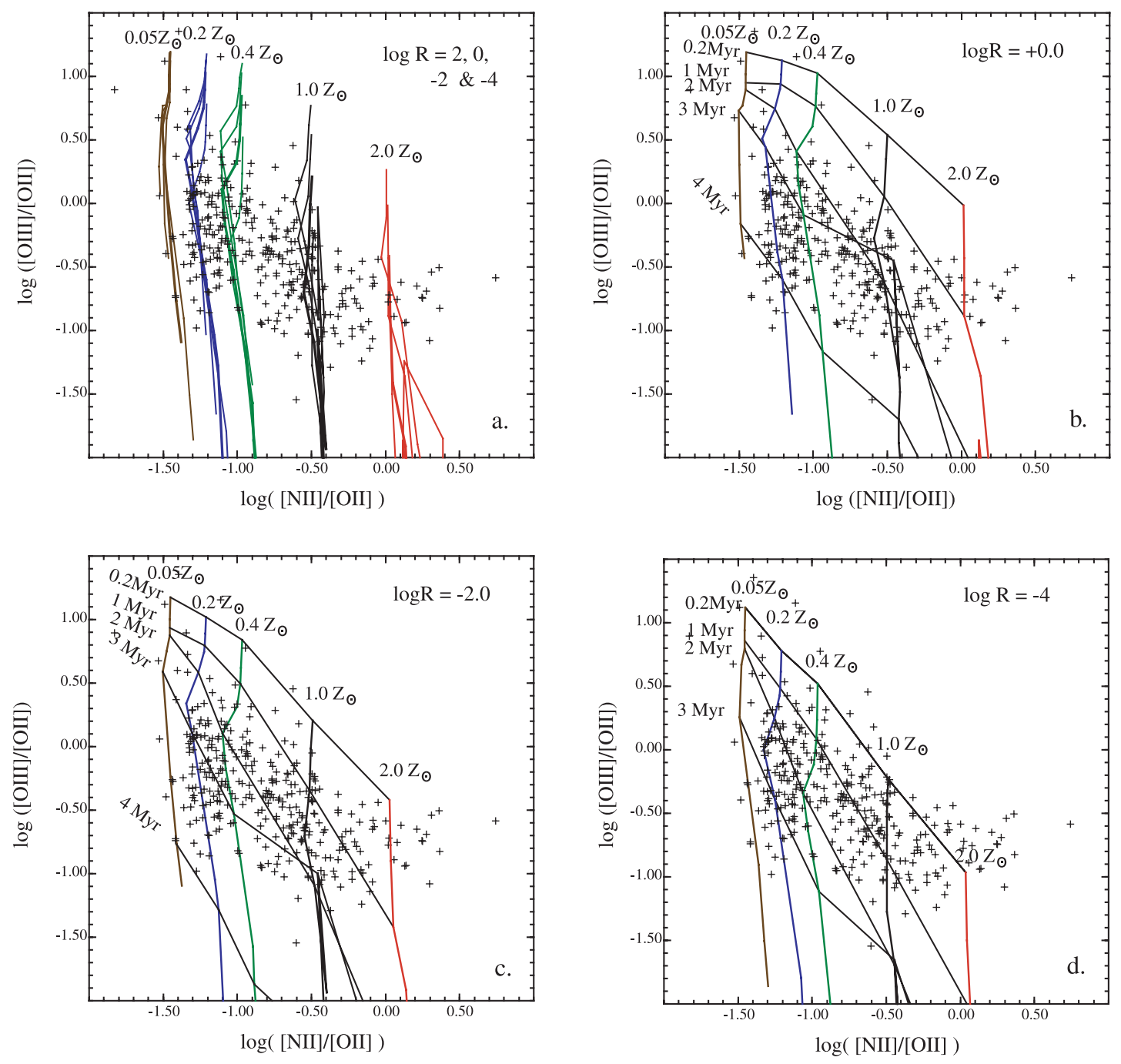

FIG. 7.-Same as Fig. 3 but for the $[\mathrm{N} \mathrm{II]} \lambda 6584 /[\mathrm{O}$ II] $\lambda \lambda 3727,3729$ ratio against the $[\mathrm{O}$ III] $\lambda 5007 /[\mathrm{O}$ II] $\lambda \lambda 3727,3729$ ratio used by Dopita et al. (2000). This is an excellent diagnostic of both metallicity and of stellar age. Note that the $[\mathrm{O}$ III] $\lambda 5007 /[\mathrm{O}$ II] $\lambda \lambda 3727,3729$ ratio is sensitive to both age and metallicity. The relationship between the observational points and the isochrones in this diagram suggests that most $\mathrm{H}$ II regions are observed in the age range 1-3 Myr.

underestimated in the models. The effect is larger than that seen in the $[\mathrm{O} \mathrm{III}] / \mathrm{H} \beta$ ratio (see Fig. 5), showing that predicted strengths of the $[\mathrm{O}$ III $]$ but especially the $\left[\mathrm{O}_{\mathrm{II}}\right]$ lines are weaker than observations suggest. This will be discussed in $\S 5$. Points with low $R_{23}$ would be explained as having metallicities greater than twice solar.

This diagnostic plot shows immediately why the calibration of the $R_{23}$ ratio in terms of metallicity has been so difficult. Not only is the range of $R_{23}$ rather restricted, but it is degenerate in terms of metallicity over a rather generous range, approximately $0.2 \leq$ $Z / Z_{\odot} \leq 1.5$. Not only that, but the ratio is fairly sensitive to excitation, as measured by the $[\mathrm{O} \mathrm{III}] /[\mathrm{O}$ II $]$ ratio. Because lowmetallicity $\mathrm{H}$ II regions are characterized by high ionization parameters, the low-metallicity points tend to occur in the upper right of this plot, while, from the form of the isochrones at high metallicity, the spray of points toward the bottom left of this diagnostic can only be due to very high metallicity $\left(Z / Z_{\odot}>2\right)$ $\mathrm{H}$ II regions with ages of 1-3 Myr.

Based on these problems, we are constrained to conclude that $R_{23}$ is not a good abundance diagnostic. We will return to this point in $\S 5$, below.

\subsubsection{Dopita et al. (2000) Diagnostics}

Dopita et al. (2000) introduced two new abundance diagnostics, based on the $[\mathrm{N}$ II] $]\left[\mathrm{O}_{\text {II }}\right]$ line ratio. This ratio was favored for two reasons that make it very sensitive to abundance. The first is that, over much of the range of interest, nitrogen is a secondary nucleosynthesis element, or at least has a large secondary component. Thus, the ratio $[\mathrm{N} \mathrm{II}] /[\mathrm{O}$ II] will systematically increase with abundance. The second is connected with the fact that the [O II] $\lambda \lambda 3727,3729$ lines are found in the near-UV part of the spectrum, whereas the [N II] $\lambda 6584$ is in the red part of the spectrum. This might be construed as a disadvantage in the use of the $[\mathrm{N} \mathrm{II}] /[\mathrm{O}$ II $]$ ratio, since the reddening corrections are large and correspondingly more uncertain. However, this problem is more than offset by the fact that the mean temperatures of $\mathrm{H}$ in regions are a strongly decreasing function of metallicity and at high abundances, the mean thermal energy of the electrons becomes too low to excite transitions with large electron excitation energy. Thus, transitions such as the $[\mathrm{O}$ II] $\lambda \lambda 3727,3729$ lines are quenched, while the lower threshold energy [ $\mathrm{N}$ II] $\lambda 6584$ continue to be excited. For this reason as well, the $[\mathrm{N} \mathrm{II}] /[\mathrm{O}$ II $]$ line ratio falls with increasing abundance.

The systematic decrease in the electron temperature with increasing metallicity may also affect the $[\mathrm{O} \mathrm{III}] \lambda 5007 /[\mathrm{O}$ II] $\lambda \lambda 3727,3729$ ratio. By itself, this would induce an increase in the $[\mathrm{O} \mathrm{III}] /[\mathrm{O}$ II] ratio with metallicity. However, this is more than offset by two factors. First, the mean effective temperature of the exciting stars falls with metallicity, and second, the mean ionization 

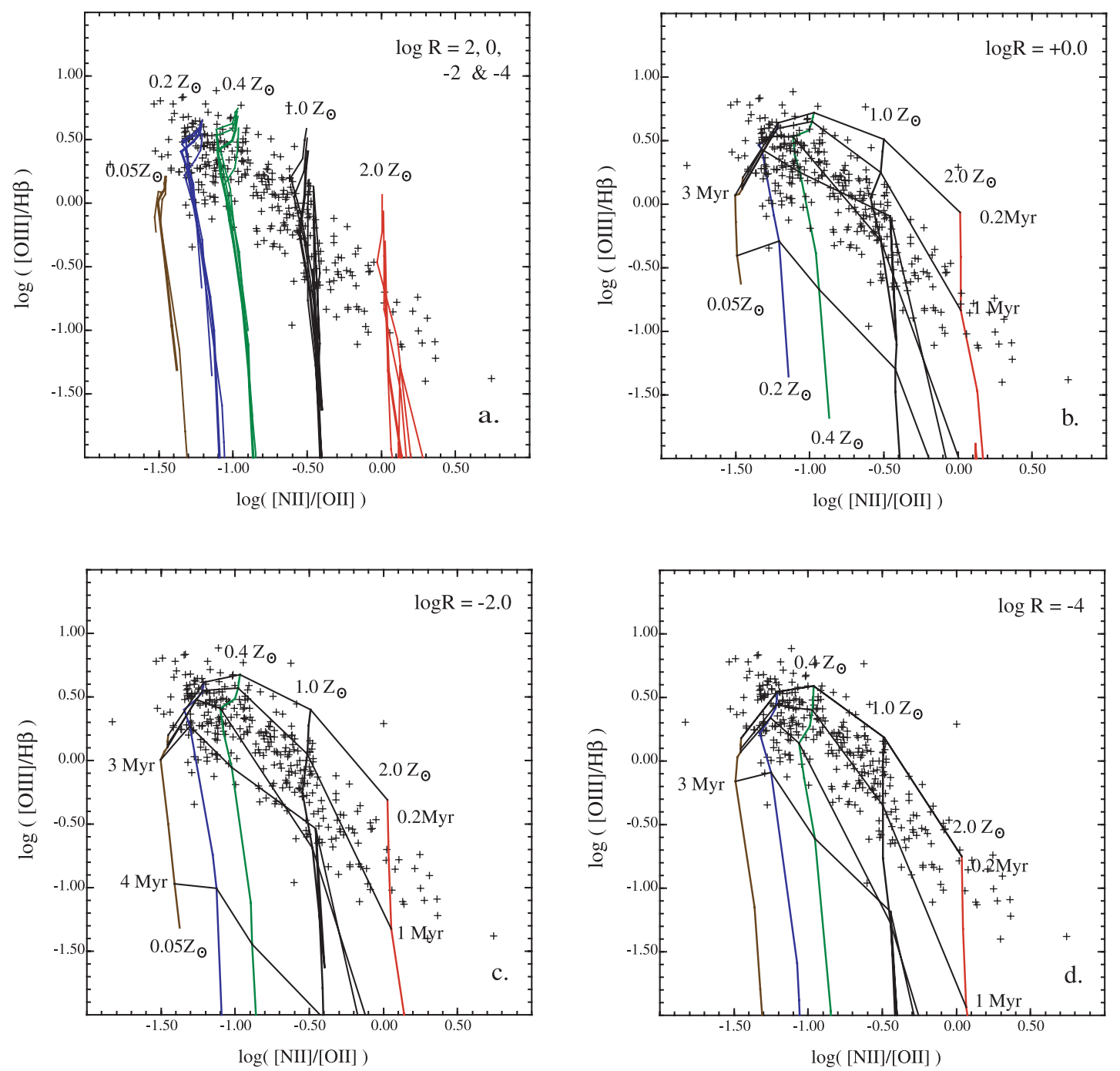

FIG. 8. - Same as Fig. 3 but for the $[\mathrm{N}$ II] $\lambda 6584 /[\mathrm{O}$ II] $\lambda \lambda 3727,3729$ ratio against the $[\mathrm{O}$ III] $\lambda 5007 / \mathrm{H} \beta$ ratio, as used by Dopita et al. (2000). This is also a good diagnostic of both metallicity and of stellar age. Given that the models tend to underestimate the $[\mathrm{O} \mathrm{m}] \lambda 5007 / \mathrm{H} \beta$ ratio, the age of the $\mathrm{H}$ II regions using this diagnostic is also likely to be an underestimate.

parameter is itself a decreasing function of metallicity. Both of these lead to a net decrease in the $\left[\mathrm{O}_{\mathrm{III}}\right] /[\mathrm{O}$ II $]$ line ratio with metallicity. Nonetheless, the $[\mathrm{O}$ III $] /[\mathrm{O}$ II $]$ line ratio remains a sensitive indicator of the ionization parameter, which is strongly age dependent. Therefore, a plot of [N $\mathrm{N}$ II $\lambda 26584 /[\mathrm{O}$ II] $\lambda \lambda 3727,3729$ against the $[\mathrm{O} \mathrm{III}] \lambda 5007 /[\mathrm{O}$ II] $\lambda \lambda 3727,3729$ ratio should separate stellar age from stellar abundance. This is illustrated in Figure 7.

It is clear that the $[\mathrm{O}$ III] $\lambda 5007 /[\mathrm{O}$ II] $\lambda \lambda 3727,3729$ ratio is an excellent diagnostic of both the stellar age and the nebular abundance. According to the theoretical tracks, all the observed H II regions have ages between 0 and roughly 4 Myr, with most lying in the age range 1-3 Myr. The division into abundance bins by use of the $\left[\mathrm{N} \mathrm{II}_{\mathrm{II}}\right] \lambda 6584 /[\mathrm{O}$ II] $\lambda \lambda 3727,3729$ ratio is very clean. Provided that the calibration is correct, then the abundance can be obtained using only the $\left[\mathrm{N}_{\mathrm{II}}\right] /[\mathrm{O} \mathrm{II}]$ ratio to a precision of roughly $0.15 \mathrm{dex}$.

The $[\mathrm{O}$ III] $\lambda 5007 / \mathrm{H} \beta$ line ratio can be substituted for the $[\mathrm{O}$ III]/ [O II] ratio, since both are sensitive to ionization parameter and therefore to the age of the cluster. However, the separation into age classes is not so sharp using this ratio, as can be seen in Figure 8 . Furthermore, we have seen in Figure 5 that the models tend to systematically underestimate the $[\mathrm{O}$ III] $\lambda 5007 / \mathrm{H} \beta$ ratio, so therefore it follows that the age of the $\mathrm{H}$ II regions inferred using this diagnostic is also likely to be an underestimate of the true age.
The third Dopita et al. (2000) diagnostic that utilizes the [N II] $\lambda 6584 /[\mathrm{O}$ II] $\lambda \lambda 3727,3729$ ratio is that which plots this ratio against the $[\mathrm{O}$ III $] \lambda 5007 /[\mathrm{N}$ II $] \lambda 6584$ ratio. The $[\mathrm{O}$ III $] \lambda \lambda 4959$, $5007 /[\mathrm{N} \mathrm{II}] \lambda \lambda 6548,6584$ ratio was first proposed by Alloin et al. (1979) as a more convenient abundance diagnostic, which is similar to the one we are using here. The observations and theoretical curves on this diagnostic are shown in Figure 9. As can be seen in the diagram, both axes are quite sensitive to abundance, but the Alloin et al. (1979) ratio, by its use of the [O III] 25007 in the place of the $[\mathrm{O}$ II] $\lambda \lambda 3727,3729$ is also more sensitive to parameters other than the abundance.

Pettini \& Pagel (2004) have argued that the [O III] $\lambda 5007 /[\mathrm{N} \mathrm{II}]$ $\lambda 6584$ ratio is of itself a sensitive abundance indicator suitable to be used in the analysis of abundances in high-redshift galaxies. This is a little too simplistic. Because the $\left[\mathrm{O}_{\mathrm{III}}\right] /[\mathrm{N}$ II $]$ ratio is strongly dependent on the excitation of the nebula, it is also sensitive to both the ionization parameter, as shown by Dopita et al. (2000) and to the age of the cluster of exciting stars. These sensitivities are as great as the sensitivity to abundance alone. Nonetheless, the [ $\left.\mathrm{N}_{\mathrm{II}}\right] \lambda 6584 /[\mathrm{O}$ II] $\lambda \lambda 3727,3729$ versus [O III] $\lambda 5007 /$ $[\mathrm{N}$ II] $\lambda 6584$ diagnostic plot has great utility in separating abundance from cluster age, and the results that it gives are in broad agreement with Figure 7 . From these two plots we can conclude 

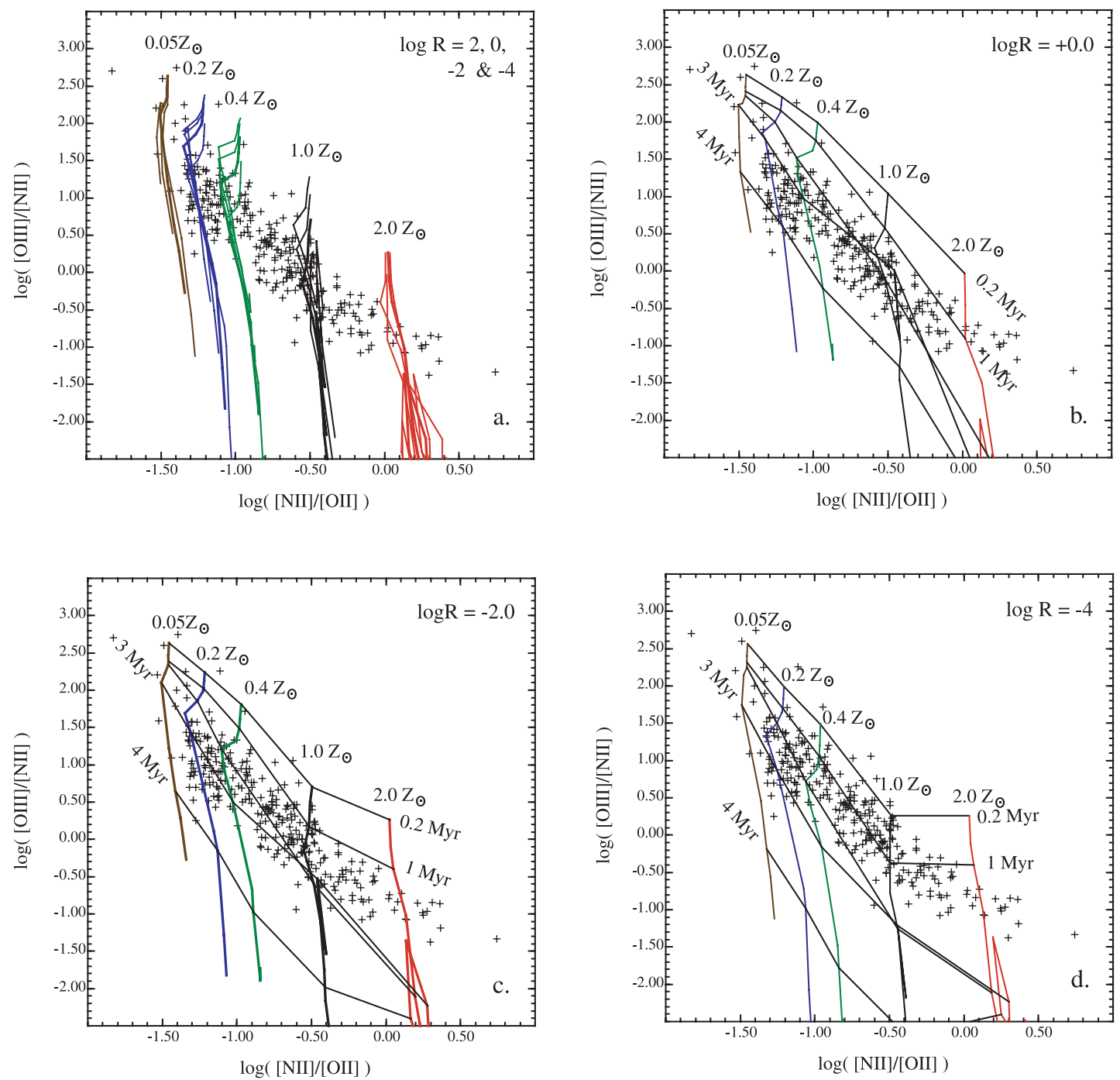

FIG. 9. - Same as Fig. 3 but for the [ $\mathrm{N}$ II] $\lambda 6584 /[\mathrm{O}$ II] $\lambda \lambda 3727,3729$ ratio against the abundance-sensitive [O III] $\lambda 5007 /[\mathrm{N}$ II] $\lambda 6584$ ratio, as proposed by Alloin et al. (1979). Although the $[\mathrm{O}$ III $] /[\mathrm{N} \mathrm{III}]$ ratio is indeed sensitive to abundance, it is also very sensitive to ionization parameter as shown by Dopita et al. (2000) and therefore to stellar age. This diagram suggests that most $\mathrm{H}$ II regions are observed in the age range 1-3 Myr, in good agreement with age estimate obtained by Fig. 7.

that most $\mathrm{H}$ II regions are observed in the age range 1-3 Myr with little sensitivity of this age range with abundance.

This is in full accord with expectations. As shown in Paper II, after an age of $3 \mathrm{Myr}$, the absolute luminosity of $\mathrm{H}$ II regions in the recombination lines drops sharply, and due to the expansion of the $\mathrm{H}$ II region, the surface brightness drops even more rapidly. Both of these militate strongly against the observation of old $\mathrm{H}$ II regions in extragalactic studies. Furthermore, very young $\mathrm{H}$ II regions tend to be dust obscured since they are still embedded in their placental molecular cloud complex. Such H II regions will not be bright at optical wavelengths. In Paper II we estimated the molecular cloud dissipation timescale for the molecular clouds to be about 1 Myr using the particular example of M17. The molecular cloud dissipation timescale was introduced in Paper I, is the characteristic timescale $\tau$ for the $\mathrm{H}$ II region to emerge from its compact or ultracompact configuration, and is a measure of the solid angle (as measured at the exciting stars) that is intercepted by molecular clouds; $\Omega(t) / 4 \pi=\exp [-t / \tau]$. Clearly, the relative dearth of young $\mathrm{H}$ II regions between 0.2 and $1.0 \mathrm{Myr}$, evident in Figures 7 and 9, is consistent with $\tau \sim 1 \mathrm{Myr}$.

In conclusion, the [ $\mathrm{N}$ II] $\lambda 6584 /[\mathrm{O}$ II] $\lambda \lambda 3727,3729$ ratio versus the $[\mathrm{O}$ III $] \lambda 5007 /[\mathrm{N}$ II] $\lambda 6584$ ratio provides a good diagnostic of both metallicity and age of the exciting cluster. The [O III] $\lambda 5007 /[\mathrm{N}$ II] $\lambda 6584$ ratio can only be used by itself as an abundance indicator to the degree to which we can assume a (fairly restricted) characteristic age for the exciting clusters. Statistically, this may be valid, but the results of its use in single galaxies should be treated with caution.

\subsection{5. $[\mathrm{N}$ II] $/ \mathrm{H} \alpha$ versus $[\mathrm{S}$ II] $/ \mathrm{H} \alpha$}

Because they lie very close to each other in wavelength, and because we have seen that $[\mathrm{N} \mathrm{II]}$ is very sensitive to abundance, the $[\mathrm{N}$ II] $\lambda 6584 / \mathrm{H} \alpha$ versus [S II] $\lambda \lambda 6717,6731 / \mathrm{H} \alpha$ plot offers some hope as an abundance diagnostic. This has recently been investigated by Moustakas \& Kennicutt (2005), who were interested to find out whether integrated spectra of galaxies were different from single $\mathrm{H}$ II regions when plotted in this way. We would expect to find such a difference in principle, because the integrated spectra do not discriminate against the emission from old $\mathrm{H}$ II regions in the manner described in the previous section. The results of our modeling is shown in Figure 10.

In Paper II, we found that the diffuse component due to old, evolved, large, and faint $\mathrm{H}$ II regions could amount to $20 \%-30 \%$ of the total $\mathrm{H} \alpha$ emission, and we suggested that this could be identified with the diffuse ionized medium (DIM), which is almost ubiquitously seen in disk galaxies (Hoopes et al. 1996; Martin 

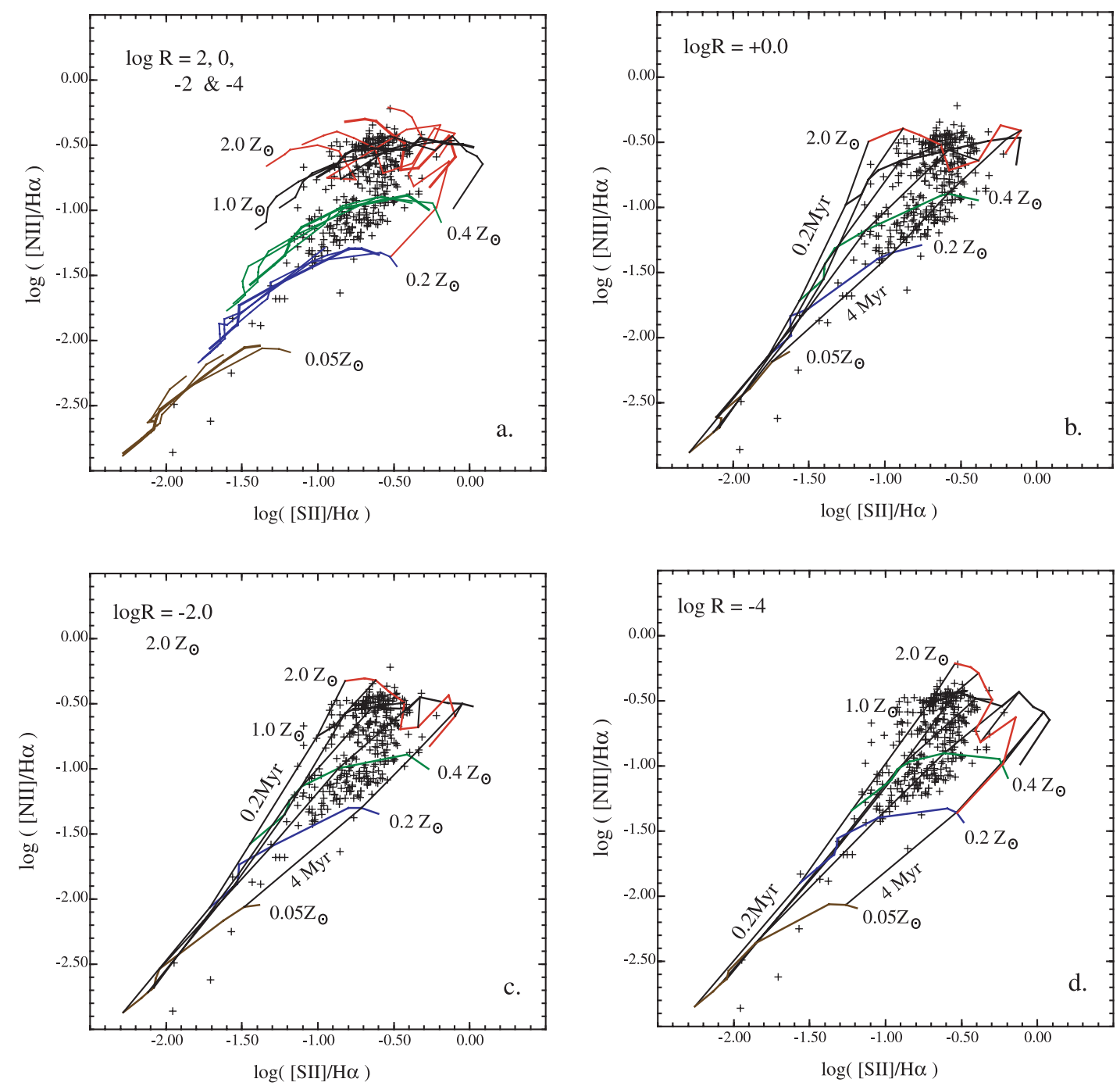

FIG. 10. - Same as Fig. 3 but for the $[\mathrm{N} \mathrm{II}] \lambda 6584 / \mathrm{H} \alpha$ vs. [S II] $\lambda \lambda 6717,6731 / \mathrm{H} \alpha$ diagnostic line ratios. As in Fig. 3 , the data for individual $\mathrm{H}$ II regions are shown as crosses, and the integrated spectra from Moustakas \& Kennicutt (2005) are shown as points. Because these are both ratios of lines in the red portion of the spectrum, and because $\mathrm{N}$ is partially a secondary element, this diagnostic turns out to be a good abundance diagnostic for $Z / Z_{\odot}<1.0$. The models of the older and more diffuse $\mathrm{H}$ iI regions are located close to the right-hand boundary of the observational points. This gas can be identified with the warm ionized medium (WIM) in galaxies.

1997; Wang et al. 1998, 1999). This is usually refereed to as the "Reynolds layer" in the context of our own Galaxy; see a recent review by Reynolds (2004). In the past, the excitation of the DIM has been usually ascribed to leakage of ionizing photons from young $\mathrm{H}$ il regions. However, the emission from large, faint, low-pressure, and evolved $\mathrm{H}$ II regions might provide a better explanation.

In Figure 10, the models of old $\mathrm{H}$ II regions with metallicities $Z / Z_{\odot}>1.0$ reach up to line ratios of $\left[\mathrm{S}_{\mathrm{II}}\right] \lambda \lambda 6717,6731 / \mathrm{H} \alpha \sim 1$ and $[\mathrm{N}$ II] $\lambda \lambda 6548,6584 / \mathrm{H} \alpha \sim 2 / 3$, which is certainly consistent with observed line ratios encountered in the DIM. However, in our Galaxy, line ratios even greater than these are seen. To explain these, we would have to invoke an additional heating mechanism, such as shocks, or a harder EUV field possibly produced by old ex-nuclei of planetary nebulae, which are sufficiently numerous to produce an appreciable EUV field out of the plane of the Galaxy.

\subsection{Infrared Line Diagnostics}

The ISO satellite first demonstrated the utility of mid-IR line diagnostics in the analysis of $\mathrm{H}$ II region spectra. For normal ga- lactic H II regions, Giveon et al. (2002) obtained extensive ISO spectroscopy, as did Peeters et al. (2002) in the context of ultracompact (UC) H II regions. Later, Verma et al. (2003) measured integrated spectra for a number of famous starburst galaxies. We will use these data here, although we note that both the highresolution and low-resolution modes of the Infrared Spectrometer (IRS; Houck et al. 2004) on Spitzer are producing much new and excellent data on both individual $\mathrm{H}$ II regions as well as on spectroscopy of whole galaxies.

Within the spectral range of the IRS, such line ratios that can be constructed are primarily sensitive to the nebular excitation. Examples include the [ $\mathrm{Ne}$ III] $15.5 \mu \mathrm{m} /[\mathrm{Ne}$ II] $12.8 \mu \mathrm{m}$ ratio, the [S IV] $10.5 \mu \mathrm{m} /[\mathrm{S}$ III] $18.7 \mu \mathrm{m}$ ratio, the [S IV] $10.5 \mu \mathrm{m} /[\mathrm{Ar}$ III] $9.0 \mu \mathrm{m}$ ratio, and the [Ne III $] 15.5 \mu \mathrm{m} /[\mathrm{S}$ III $] 18.7 \mu \mathrm{m}$ ratio. Unfortunately, these are of little use in measuring anything other than the hardness of the EUV radiation field. All of the strong lines accessible in this region of the spectrum are produced by $\alpha$-process elements, and therefore the abundance ratios stay approximately constant with abundance. All the lines have lowenergy thresholds for excitation, and therefore none of them are particularly sensitive to the electron temperature, which varies 


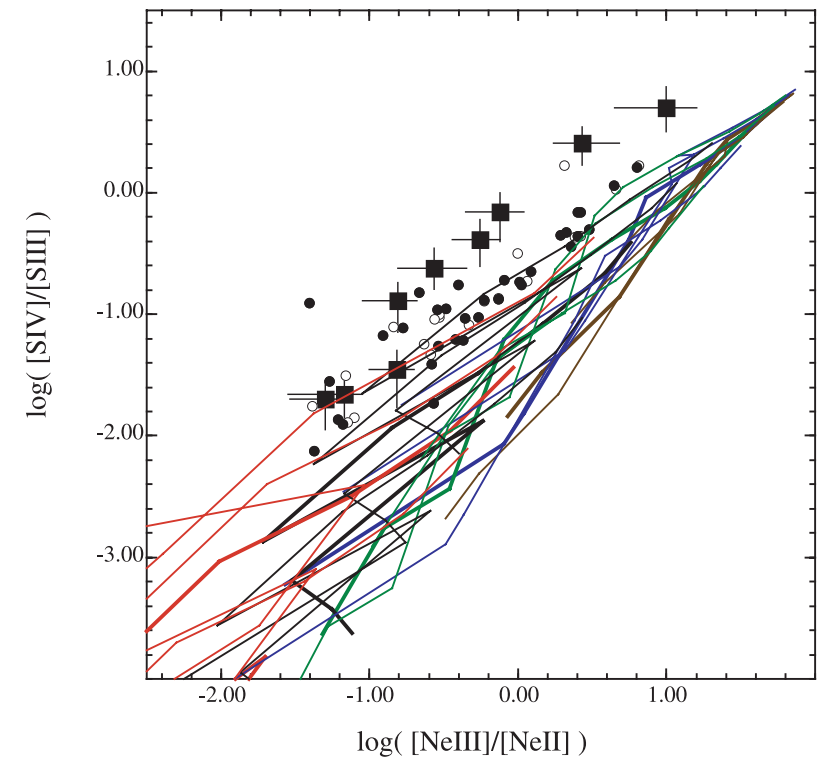

FIG. 11 . -The [Ne III] $15.5 \mu \mathrm{m} /[\mathrm{Ne}$ II] $12.8 \mu$ m ratio vs. the [S IV] $10.5 \mu \mathrm{m} /$ [S III] $18.7 \mu \mathrm{m}$ ratio. H II regions from Giveon et al. (2002) are plotted as filled circles, UC H II regions from Peeters et al. (2002) as open circles, and starburst galaxies from Verma et al. (2003) as filled squares with error bars. The theoretical curves create as superb an example of theoretical spaghetti as anything these authors have seen.

strongly with abundance. In Figure 11 we present only one example to demonstrate how poor these line ratios are as abundance diagnostics when used alone.

Much of the data used in this plot refers to observations of ultracompact $\mathrm{H}$ II regions, which are not exactly comparable to "normal" $\mathrm{H}$ II regions. In particular, they are extremely dense, most are excited by only a single OB star, and many of the ionizing photons from the exiting star are absorbed by dust rather than by the ionized plasma. This accounts for much of the offset between the (low-density) models and the data in Figure 11. This effect is particularly important for the [S IV] $10.5 \mu \mathrm{m} /\left[\mathrm{S}_{\text {III }}\right] 18.7 \mu \mathrm{m}$ ratio. These points, and this particular diagnostic plot, was thoroughly discussed in our paper on compact H II regions (Dopita et al. 2006a).

In order to form a line ratio that is sensitive to abundance, we need to use a ratio with a hydrogen or helium recombination line. Unfortunately, these are rather faint in the mid-IR. About the best line we have is $\operatorname{Br} \alpha 4.051 \mu \mathrm{m}$. Alternatively, Pfund $-\alpha 7.458 \mu \mathrm{m}$ might be usable, although it is often difficult to observe against the PAH features seen at this wavelength. In Figure 12 we show one such diagnostic, the [Ne III] $15.5 \mu \mathrm{m} /[\mathrm{Ne}$ II] $12.8 \mu \mathrm{m}$ ratio versus the [Ne III] $15.5 \mu \mathrm{m} / \mathrm{Br} \alpha 4.051 \mu \mathrm{m}$ line ratio. This has the advantage that, at a given value of the excitation measured by the [Ne III] $15.5 \mu \mathrm{m} /[\mathrm{Ne}$ II] $12.8 \mu \mathrm{m}$ ratio, the [Ne III] $15.5 \mu \mathrm{m} /$ $\operatorname{Br} \alpha 4.051 \mu \mathrm{m}$ line ratio is a monatonic function of abundance up to at least $Z \sim 2.0 \times Z_{\odot}$, and so it provides a clean abundance diagnostic, unlike the comparable optical line ratio shown in Figure 5.

Currently, the uncertainties in the observational data are to large for this to provide a very accurate abundance diagnostic. However, we may note that the observations from Giveon et al. (2002) are consistent with abundances between 2.0 and $0.4 Z_{\odot}$, and with ages between 0 and $2 \mathrm{Myr}$. This abundance range is exactly what we would expect to find for Galactic $\mathrm{H}$ in regions over the range of Galactocentric radii observed by them. It is interesting that the ages are lower than those derived for $\mathrm{H}$ II regions at optical wavelengths. This is consistent with the selection criteria for IR-bright,

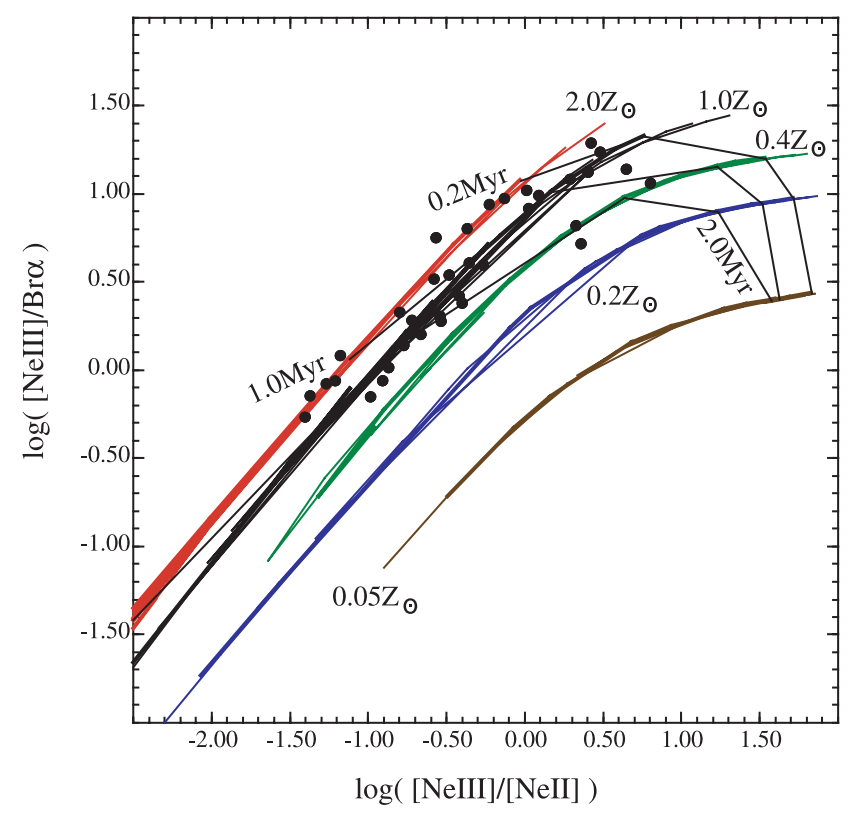

FIG. 12.-The [Ne III] $15.5 \mu \mathrm{m} /[\mathrm{Ne}$ II] $12.8 \mu \mathrm{m}$ ratio vs. the [Ne III] $15.5 \mu \mathrm{m} /$ $\operatorname{Br} \alpha 4.051 \mu \mathrm{m}$ line ratio. H II regions from Giveon et al. (2002) are plotted as filled circles. This diagnostic provides a clean abundance determination that is monotonic over the full abundance range. The isochrones are given for the case $\log \mathcal{R}=-2$. This diagram is an infrared analog of Fig. 5 but is a much cleaner diagnostic because these IR line ratios are very insensitive to the nebular temperature.

compact $\mathrm{H}$ II regions. This selects for young $\mathrm{H}$ II regions. In addition, the use of mid-IR wavelengths allow the $\mathrm{H}$ II region emission to be seen almost unimpeded by the surrounding molecular material, so the molecular cloud dissipation timescale is not a relevant parameter here.

\section{INTEGRATED SPECTRA}

The diagnostics presented in the previous sections were designed to derive parameters for single $\mathrm{H}$ II regions. However, what we seek to do now is to develop a spectral synthesis by taking recombination line flux-averaged spectra along an evolutionary track of an $\mathrm{H}$ in region with given parameters. This reduces the number of free parameters to two: $Z / Z_{\odot}$ and $\log \mathcal{R}$. The observations can then be used to constrain the values of $\log \mathcal{R}$, allowing the extragalactic abundance sequence for ensemble averages of evolving $\mathrm{H}$ II regions to be derived. This technique opens the way to the accurate study of the metallicity evolution of both individual galaxies and the universe.

The model we adopt assumes that clusters are formed continuously and that any single cluster has all of its stars born coevally. As described above, the cluster mass function does not enter explicitly into the models, which are characterized by their $R$-values. The process of producing an integrated spectrum for any $Z$ is therefore simply to integrate the fluxes from a single $\mathrm{H}$ II model through time, until it has faded such that $\geq 96 \%$ of all ionizing photons have been emitted. This limits the integration time to lie within the first 5-6.5 Myr. Stochastic effects due to finite sampling of the stellar IMF-important at low cluster massesare not included. However, the form of the cluster IMF either as inferred in Paper II or derived from stellar number counts is such that each logarithmic bin of cluster mass contributes a similar amount to the total flux. Massive clusters will therefore tend to wash out the stochastic effects of low-mass clusters.

For line ratio diagnostics, there is no simple relationship between the ensemble-averaged line ratio and the line ratios in the 


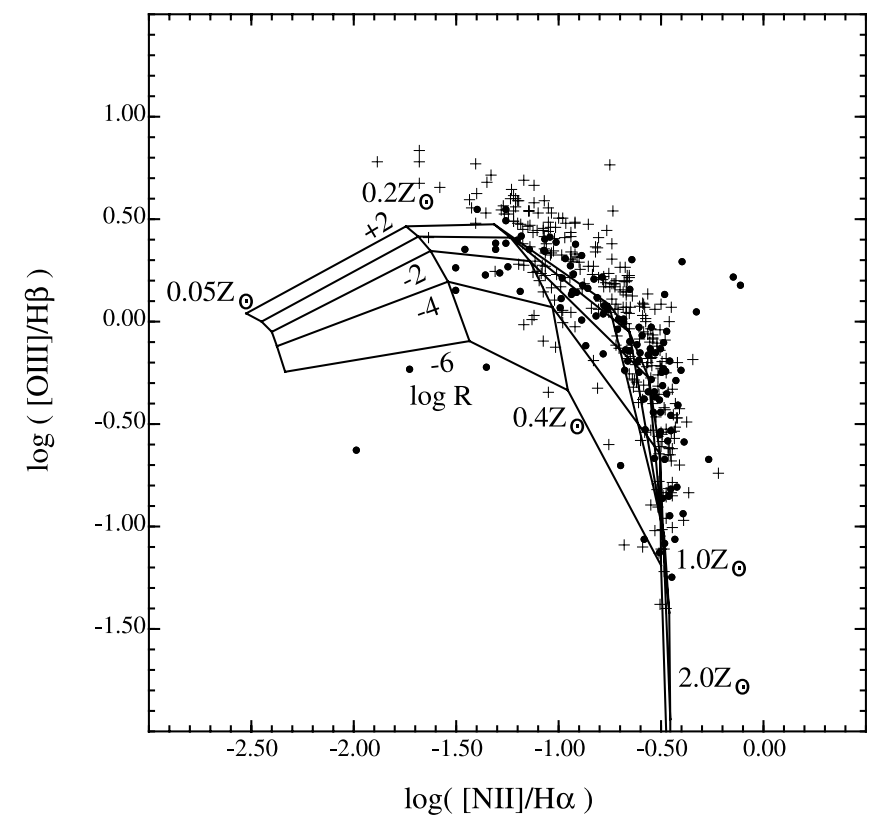

FIG. 13.-Synthesized H II region spectra on the V\&O diagnostic [N II] $\lambda 6584 / \mathrm{H} \alpha$ vs. [O III ] $\lambda 5007 / \mathrm{H} \beta$ (cf. Fig. 3 ). The integrated galaxy spectra from the Nearby Field Galaxy Survey (NFGS; Jansen et al. 2000) are shown as filled circles, while the crosses represent individual $\mathrm{H}$ in regions drawn from the sources referenced above, to show the degree to which the integrated spectra differ from the single source spectra. Note the large disparity between the integrated spectra and the observations of individual $\mathrm{H}$ II regions at the low-abundance end $\left(Z / Z_{\odot}<0.4\right)$. Compared with earlier models, the theory provides a good fit to the integrated galaxy data, providing that $\log \mathcal{R}>-4$, and emphasizes the degeneracy of this $\mathrm{V} \& \mathrm{O}$ diagnostic.

individual $\mathrm{H}$ II regions that make up this average. For example, a high ionization line such as [O III] $\lambda 5007$ will be mostly produced in the very youngest $\mathrm{H}$ II regions with the highest $R$-values. Low ionization lines such as the $[\mathrm{S}$ II] $\lambda \lambda 6717,6731$ lines will have a much greater weighting from older evolved $\mathrm{H}$ in regions with low $R$-values. Indeed, for this line the majority of the line flux may come from regions lying outside the bright $\mathrm{H}$ in regions that would be normally selected for observation in studies of individual $\mathrm{H}$ II regions - in other words, from regions that form part of the "warm ionized medium" (WIM).

Given these quite different selection biases, it is interesting to compare line ratio observations of the integrated line fluxes of galaxies with those of individual $\mathrm{H}$ in regions. Such a comparison helps us to quantify the importance of the WIM in determining the global line ratio. For this purpose, we have used the observations of individual $\mathrm{H}$ II regions listed and used above (Fig. 3 and references in $\S 3.1$ ), and we have drawn our sample of integrated line fluxes of galaxies from the Nearby Field Galaxy Survey (NFGS; Jansen et al. 2000), and the recent survey by Moustakas $\&$ Kennicutt (2005).

In Figure 13 we plot the main V\&O diagnostic [ $\left.\mathrm{N}_{\text {II }}\right] \lambda 6584 / \mathrm{H} \alpha$ versus [O III] $25007 / \mathrm{H} \beta$. This should be compared with Figure 3. The models provide a good fit to the integrated spectra of galaxies. Indeed, the fit is better than that of the models of individual H II regions compared to data in Figure 3. This is especially apparent at the low-abundance end (where most observations are of relatively nearby dwarf irregular galaxies such as NGC 6822, IC 1613, II $\mathrm{Zw} 40$, and the like). This suggests that the observations of these low-abundance $\mathrm{H}$ II regions may be affected by aperture effects. This would be caused by observers picking out bright highexcitation subregions of the $\mathrm{H}$ II region from their long-slit spectra for analysis, rather than observing across the whole $\mathrm{H}$ II region

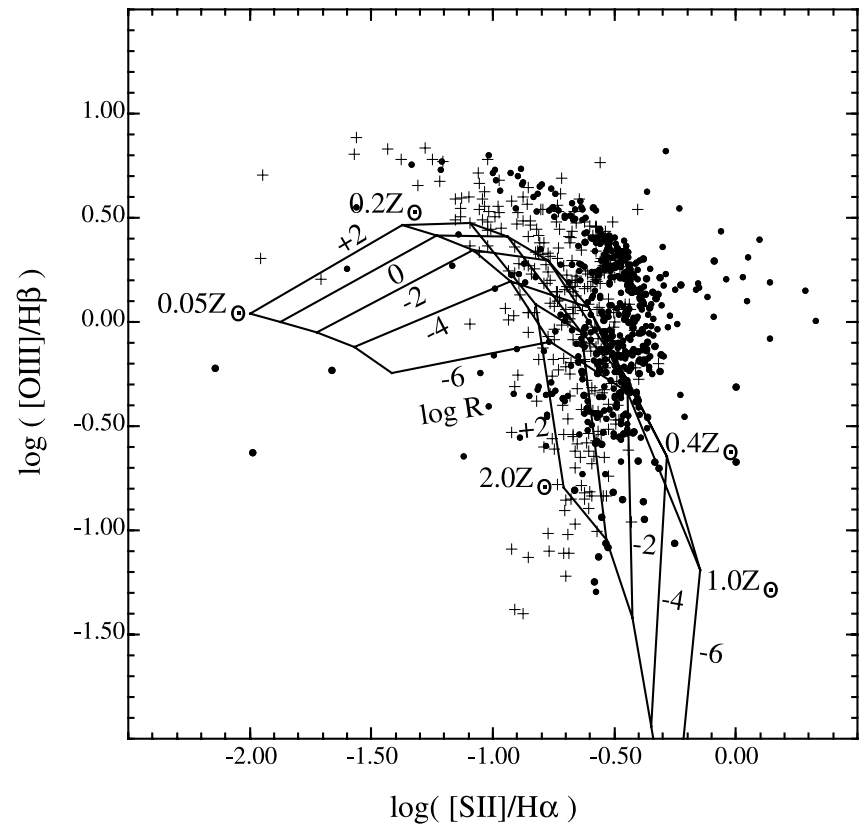

FIG. 14.- Synthesized H II region spectra on the V\&O diagnostic [S II] $\lambda \lambda 6717$, $6731 / \mathrm{H} \alpha$ vs. [O III] $\lambda 5007 / \mathrm{H} \beta$ (cf. Fig. 5). The integrated galaxy spectra are shown as filled circles and include both the NFGS data and the data from Moustakas \& Kennicutt (2006). Both the [S II] $\lambda \lambda 6717,6731$ and [O III] $\lambda 5007$ lines seem to be a little weak in the models compared with the observations.

to obtain an integrated spectrum of it. This would preferentially enhance the $\left[\mathrm{O}_{\mathrm{III}}\right] \lambda 5007 / \mathrm{H} \beta$ ratio while at the same time depressing the $[\mathrm{N}$ II $] \lambda 6584 / \mathrm{H} \alpha$ ratio, moving the observed point in line ratio space toward the top and the left.

The fit with the second $\mathrm{V} \& \mathrm{O}$ diagnostic, the $[\mathrm{S}$ II] $\lambda \lambda 6717$, $6731 / \mathrm{H} \alpha$ versus [O III] $\lambda 5007 / \mathrm{H} \beta$ (Fig. 14) is less convincing. The integrated spectra are displaced to the right on the [S II] $\lambda \lambda 6717,6731 / \mathrm{H} \alpha$ ratio compared with the spectra from individual $\mathrm{H}$ II regions, showing the importance of the contribution of the more diffuse ionized component. It is interesting to note that most of the highest points in terms of the [O III] $25007 / \mathrm{H} \beta$ ratio are contributed by the Moustakas \& Kennicutt (2005) data set. In this respect there are systematic differences between this data set and the NFGS survey. The reason for this is not apparent to us.

In the models, the $\left[\mathrm{N}_{\mathrm{II}}\right] \lambda \lambda 6548,6584$ are the major coolants in the outer parts of the $\mathrm{H}$ II regions. It is possible that the abundance of nitrogen in the ionized gas is somewhat overestimated (by an underestimate of the depletion, for example), leading to saturation in the $[\mathrm{N} \mathrm{II}] \lambda 6584 / \mathrm{H} \alpha$ ratio, and a reduction in the [S II] $\lambda \lambda 6717,6731 / \mathrm{H} \alpha$ ratio. The problem with the models in reproducing the observed $[\mathrm{S}$ II] $\lambda \lambda 6717,6731 / \mathrm{H} \alpha$ ratios is systematic. Even with the old Starburst99 version 2 models, a similar problem was encountered by Dopita et al. (2000). This will be discussed further in the next section.

Let us now turn our attention to the BPT diagram, the [O III] $\lambda 5007 /[\mathrm{O}$ II $] \lambda \lambda 3727,3729$ ratio versus the $[\mathrm{O}$ III $] \lambda 5007 / \mathrm{H} \beta$ ratio (Baldwin et al. 1981). This is plotted in Figure 15. Once again, the fit between the theory and observation is good, although on this diagnostic the data once again fall somewhat high relative to the theory in the $[\mathrm{O}$ III $] \lambda 5007 / \mathrm{H} \beta$ ratio at the low-abundance end $Z / Z_{\odot}<0.4$.

We note that the $\left[\mathrm{N}_{\mathrm{II}}\right] \lambda 6584 / \mathrm{H} \alpha$ ratio covers quite a large range and displays considerable sensitivity to abundance. However, the same is not true of the $[\mathrm{O}$ III] $\lambda 5007 / \mathrm{H} \beta$ ratio. Only two points in the NFGS survey lie below $\log [\mathrm{N}$ II] $\lambda 6584 / \mathrm{H} \alpha=$ -1.5 , and since the $Z / Z_{\odot}$ curve lies to the left of $\log [\mathrm{N}$ II] 


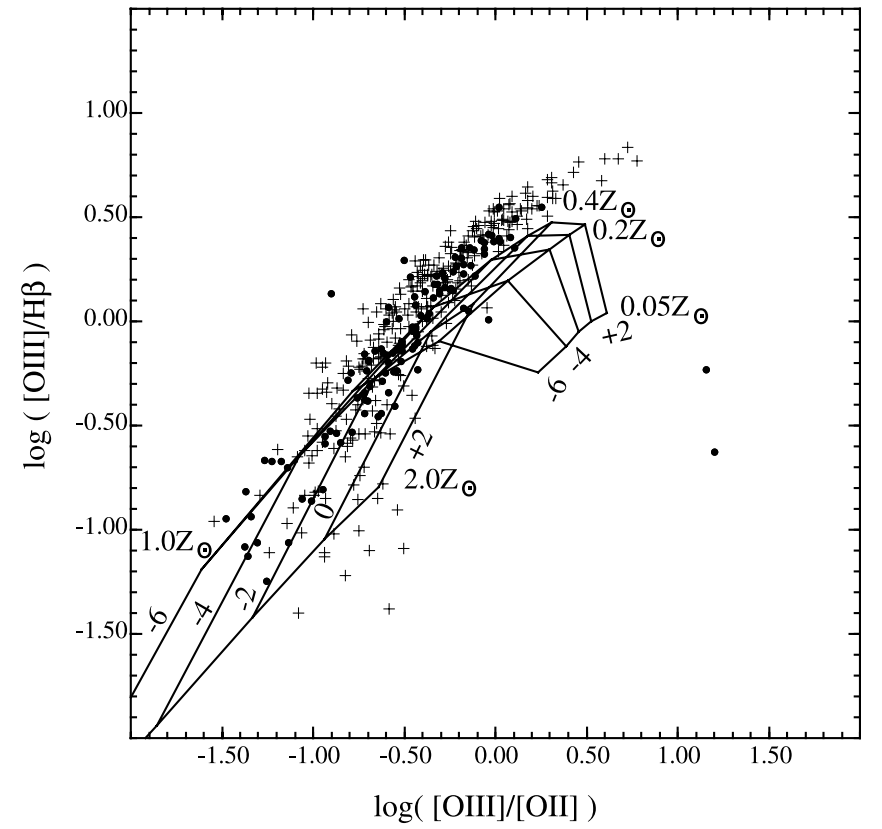

FIG. 15.-Same as Fig. 13, but for the BPT diagram, the [O III] $25007 /[\mathrm{O}$ II] $\lambda \lambda 3727,3729$ ratio vs. [O III] $\lambda 5007 / \mathrm{H} \beta$. This should be compared with Fig. 5 .

$\lambda 6584 / \mathrm{H} \alpha=-1.5$, Figure 13 suggests that the lower abundance bound to the NFGS survey is about $Z / Z_{\odot}<0.2$. This is also consistent with what would be implied from Figure 15. Both Figure 14 and Figure 15 suggest that the upper bound is about $Z / Z \sim 2$.

The first of the Dopita et al. (2000) diagnostics is shown in Figure 16. This plots the [N $\mathrm{II}] \lambda 6584 /[\mathrm{O}$ II $] \lambda \lambda 3727,3729$ ratio against the $\left[\mathrm{O}_{\mathrm{III}}\right] \lambda 5007 /[\mathrm{O}$ II $] \lambda \lambda 3727,3729$ ratio and should be compared with Figure 7 . There is very little distinction between the distribution of the integrated spectra from the NFGS survey and the observations of individual $\mathrm{H}$ II regions on this diagram. Essentially, any value of $\log \mathcal{R}$ seems to be permitted, although the majority of the points are consistent with a range from 0 to -4 in this parameter. This is consistent with what we might have expected, given that the parameters that enter into $R, M_{\mathrm{cl}}$, and $P / k$ cover at least the range $100<M_{\mathrm{cl}} / M_{\odot}<10^{5}$ and $10^{4} \mathrm{~cm}^{-3} \mathrm{~K}<$ $P / k<10^{7} \mathrm{~cm}^{-3} \mathrm{~K}$.

The abundance range of the NFGS survey suggested by this plot is broader than that given by the V\&O or BPT diagnostics, since the theory implies the existence of some galaxies with very low abundances. However, we must beware once again of the observational problems that enter into plots like this. The [N II] $\lambda 6584$ line and the $[\mathrm{O}$ II] $\lambda \lambda 3727,3729$ lines lie at opposite ends of the spectrum, and so there is a very large reddening correction applied to the data. Generally speaking, a standard reddening law is used to do this. In the case of the NFGS survey, for example the Clayton et al. (1989) extinction curve was used. However, the foreground dust screen in front of an individual $\mathrm{H}$ II region (let alone a whole galaxy) has a complex fractal structure, and rather than using an extinction curve, an attenuation curve should be used instead, see Fischera et al. (2003), Fischera \& Dopita (2005), and Paper I.

The effect of an attenuation law will be to cause the strengths of lines in the red to be underestimated, while UV lines such as the [O II] $\lambda \lambda 3727,3729$ lines will be systematically overestimated. These effects will mean that the observations corrected using an extinction law will land systematically too far to the left and too low on this diagram, while on (almost) extinction-free

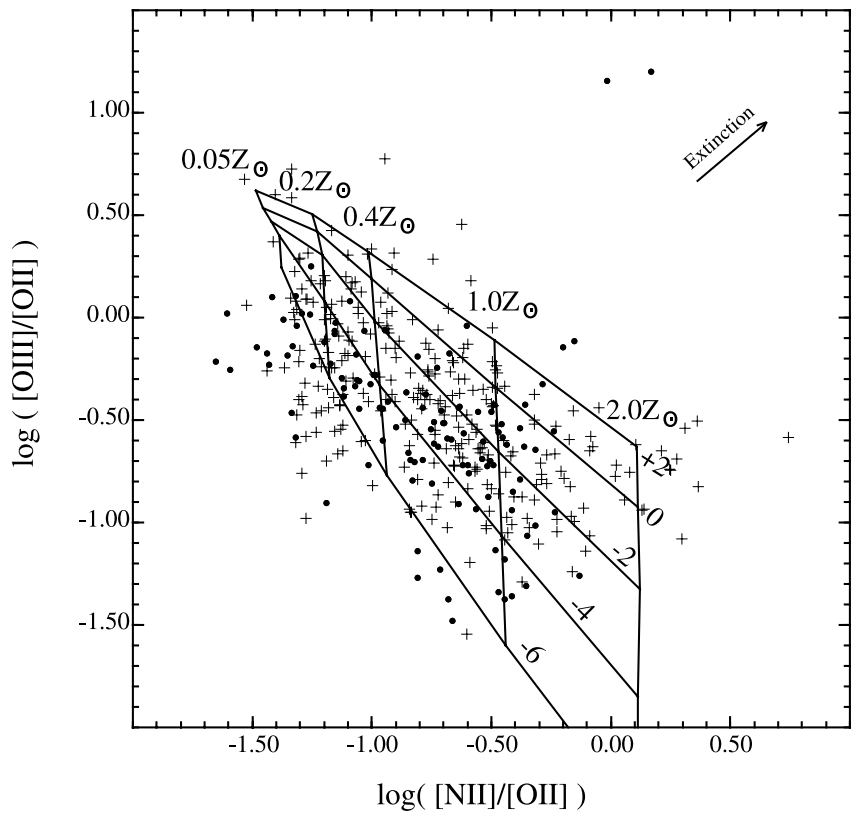

FIG. 16.-Same as Fig. 13 but for the [N II] $\lambda 6584 /[\mathrm{O}$ II] $\lambda \lambda 3727,3729$ ratio against the $\left[\mathrm{O}\right.$ III] $\lambda 5007 /\left[\mathrm{O}_{\mathrm{II}}\right] \lambda \lambda 3727,3729$ ratio used by Dopita et al. (2000). This should be compared with Fig. 7. This is perhaps the most unambiguous diagnostic for the determination of both metallicity and $\log \mathcal{R}$. For the meaning of the arrow labeled "extinction," see the text.

diagnostics such as the V\&O plots the data will plot onto the correct position. If this effect is the cause of the displacement between theory and observation seen in Figure 15, this suggests that the $[\mathrm{O}$ III $] \lambda 5007 /[\mathrm{O}$ II $] \lambda \lambda 3727,3729$ ratio may be systematically underestimated by $\sim 0.3$ dex. This would imply that the $[\mathrm{N} \mathrm{II}]$ $\lambda 6584 /[\mathrm{O}$ II] $\lambda \lambda 3727,3729$ ratio may be underestimated by a similar amount, since the extinction is determined from the $\mathrm{H} \alpha / \mathrm{H} \beta$

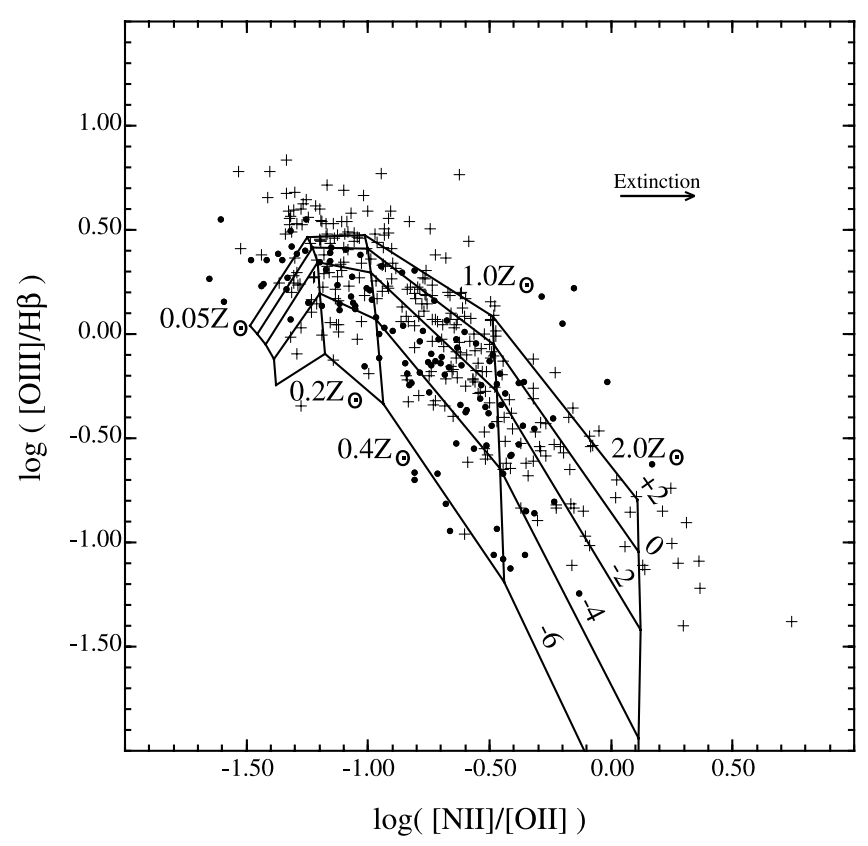

FIG. 17.- Same as Fig. 13, but for the [N II] $\lambda 6584 /\left[\mathrm{O}_{\text {II }}\right] \lambda \lambda 3727,3729$ ratio against the $[\mathrm{O}$ III $] \lambda 5007 / \mathrm{H} \beta$ ratio used by Dopita et al. (2000). This should be compared with Fig. 8. This is a good diagnostic for the determination of both metallicity and $\log \mathcal{R}$. The observations and the theory would give consistent answers in all integral spectrum diagnostics if a systematic correction is made to the observations of the magnitude illustrated by the arrow labeled "extinction" in this and the previous figure (see text). 


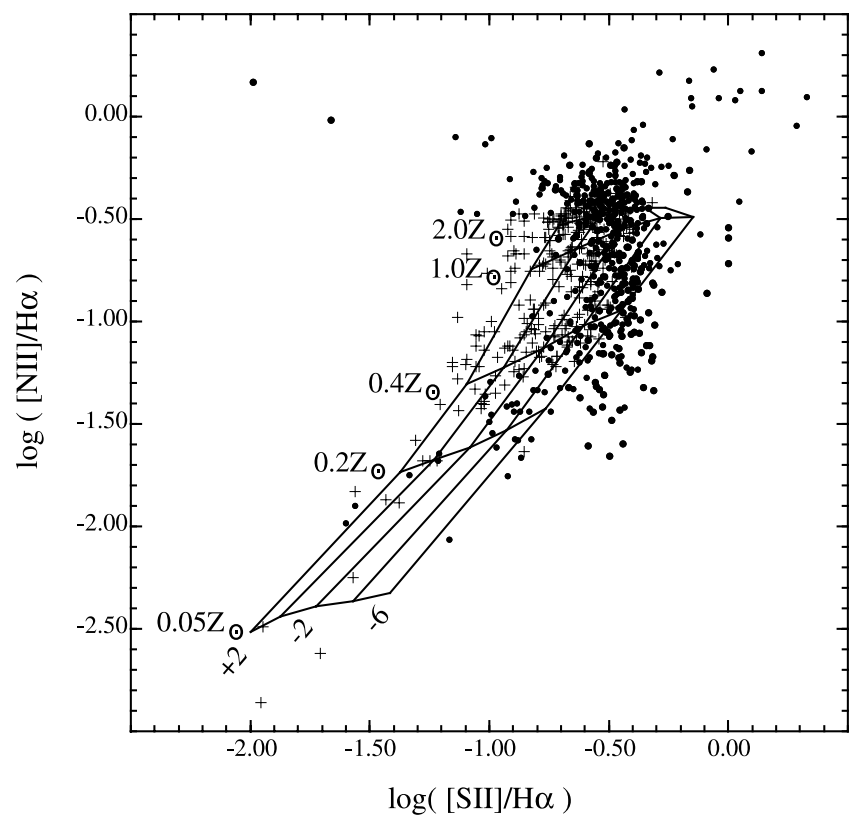

FIG. 18.-Same as Fig. 13, but for the [N II] $\lambda 6584 / \mathrm{H} \alpha$ ratio against the [S II] $\lambda \lambda 6717,6731 / \mathrm{H} \alpha$ ratio. This should be compared with Fig. 10. This once again illustrates our problems in modeling the $\left[\mathrm{S} \mathrm{II}_{\mathrm{II}}\right.$ line strengths.

ratio, which means that the observed [N II] $\lambda 6584 /[\mathrm{O}$ III] $\lambda 5007$ ratio must necessarily be correct. The effect of a correction of this magnitude is shown on Figure 16 as an arrow labeled "extinction." With a correction of this magnitude, not only can the lower bound of the abundance in the NFGS survey be reconciled with that given by Figure $13\left(Z / Z_{\odot} \sim 0.2\right)$, but also with the upper bound indicated by Figure $15\left(Z / Z_{\odot} \sim 2\right)$. The inferred range on $\log \mathcal{R}$ would then be from 2 to -4 .

A systematic overestimate of the [O II] $\lambda \lambda 3727,3729$ lines would also lead to a systematic overestimate of $R_{23}$. This may well account for much of the discrepancy between theory and observation in Figure 6.

A similar effect is seen in the second of the Dopita et al. (2000) diagnostics is shown in Figure 17. This plots the [N $\left.\mathrm{N}_{\mathrm{II}}\right] \lambda 6584 /\left[\mathrm{O}_{\mathrm{II}}\right]$ $\lambda \lambda 3727,3729$ ratio against the $[\mathrm{O}$ III $] \lambda 5007 / \mathrm{H} \beta$ ratio. Once again, a systematic extinction correction error of 0.3 dex would shift the observational points into a range that is consistent with the both the abundance and $R$ bounds inferred above.

Finally, we plot the $[\mathrm{S}$ II] $\lambda \lambda 6717,6731 / \mathrm{H} \alpha$ ratio versus the $[\mathrm{N}$ II] $\lambda 6584 / \mathrm{H} \alpha$ line ratio in Figure 18 (compare with Fig. 10). This shows, once again, the value of the $[\mathrm{N} \mathrm{II}] \lambda 6584 / \mathrm{H} \alpha$ line ratio as an abundance diagnostic in its own right. It also illustrates, once again, our difficulties with the $[\mathrm{S}$ II] $\lambda \lambda 6717,6731 / \mathrm{H} \alpha$ ratio, with the observations, particularly the Moustakas \& Kennicutt (2005) data set falling to the right of the theoretical curves in this ratio.

Note how the integrated spectra on Figure 18 are concentrated along the right-hand side of the region defined by the observations of single $\mathrm{H}$ II regions. This reflects the fact that integrated galaxy spectra include the diffuse component of the ISM, even when this has a low surface brightness, because the total flux in the diffuse component may be an appreciable portion of the total emission. Indeed, a recent study by Oey et al. (2006) suggests that the "diffuse" fraction may exceed 50\% in nearby starburst galaxies.

\section{DISCUSSION AND CONCLUSIONS}

It should now be clear that these models represent a significant advance in our understanding of the strong line spectra of $\mathrm{H}$ II regions. In particular, the models explicitly account for the dynamical evolution of the $\mathrm{H}$ II region controlled through the mechanical energy input by both the stellar winds and supernova explosions. Two factors largely control the excitation of the young $\mathrm{H}$ II regions, both of these dependent on the chemical abundance:

1. The effective temperature of the exciting stars, which increases with decreasing metallicity.

2. The control of the ionization parameter by the stellar winds in the expanding $\mathrm{H}$ II regions. This was shown in Paper II to increase with decreasing metallicity.

Since both of these effects operate to increase the excitation of low-abundance $\mathrm{H}$ in regions, we therefore find an abundance dependent excitation, which much improves the fit of the theory compared to models in which the ionization parameter is treated as a free variable. In our models the ionization parameter is replaced by the $R$ parameter, the ratio of the cluster mass in solar masses to the pressure in the ISM; $P / k\left(\mathrm{~cm}^{-3} \mathrm{~K}\right)$. This parameter is used because all models with a given $R$ have a unique relationship between their instantaneous ionization parameter and time. This allows us to construct evolutionary tracks for ensembles of $\mathrm{H}$ II regions.

With increasing age, the ionization parameter falls almost monotonically, and the $\mathrm{H}$ II region fades after reaching its maximum brightness in $\mathrm{H} \alpha$ after 1-2 Myr. The presence of Wolf-Rayet stars after about 3.5 Myr causes the effective temperature of the cluster to briefly increase. This increases the excitation for a short time, causing the 3 and the 4 Myr isochrones to cross over each other on diagnostic plots for the cases of high metallicity $\left(Z / Z_{\odot}>0.5\right.$, approximately).

In this paper, we have identified a number of useful diagnostics for deriving the abundance and either the age, in the case of isolated $\mathrm{H}$ II regions, or the $R$ parameter, in the case of integrated spectra of galaxies. Our diagnostics for the integrated spectra are the first that explicitly take into account the fact that when we observe a whole galaxy, we are observing an ensemble average of $\mathrm{H}$ II regions of all ages, sizes and central cluster masses. The simplification we have been able to make by using the $R$ parameter has rendered this problem tractable to a more simple analysis other than treating each $\mathrm{H}$ II region separately and adding the whole lot together at the end.

In comparing the models to the observational data we have identified some potential problems with the way that observations of $\mathrm{H}$ II regions have been made and analyzed.

1. Long-slit observations do not integrate over the whole of an $\mathrm{H}$ II region, particularly in the case of observations of nearby, low-metallicity dwarf irregular galaxies. By picking out the brightest regions of such $\mathrm{H}$ II regions, we bias the data toward the high-excitation regions and the spectrum is not representative of the whole H II region (Luridiana \& Peimbert 2001; Stasinska \& Izotov 2003).

2. The use of a reddening correction using an extinction curve, rather than an attenuation curve (which is appropriate for spatially extended objects), will cause the line intensities of UV lines to be systematically overestimated, biasing the strong line diagnostics that use the $[\mathrm{O}$ II] $\lambda \lambda 3727,3729$ lines.

This second effect would reveal itself in the spectrophotometry as a failure to reproduce the correct intensities of the higher members of the Balmer series, such as $\mathrm{H} \delta$ or $\mathrm{H} \epsilon$, and so should be quite easy to check for in observational data sets. Such an effect would also influence the measurement of the strength of the 
[O III] $\lambda 4363$ line, again causing it to be overestimated. This could be part of the reason for the long-standing discrepancy between the abundances derived from the strong lines and those obtained from measurements of the electron temperature using ratios such as [O III] $\lambda 4363 /[\mathrm{O}$ III] $\lambda 5007$; see Bresolin et al. (2004) for a recent discussion. We expect that the major part of the discrepancy will turn out to be caused by temperature fluctuations. However, this could be easily corrected for by assuming the Case B recombination line ratio between $\mathrm{H} \beta$ and $\mathrm{H} \gamma$ and measuring the forbidden line strengths relative to the nearest Balmer lines in the spectrum.

There is clearly a need for integral field spectrophotometry on extragalactic $\mathrm{H}$ II regions. This would allow efficient construction of integrated spectra and assist in the removal of stellar flux from the spectrum. Such observations would not only help to remove the remaining discrepancies between theory and observation, but also give insight into the use of theoretical integral spectra in the analysis of the chemical evolution history of the early universe.

Our models have their deficiencies. In general, all the lowionization species are predicted weaker than observed. These include the [O I] $\lambda 6300$, the [S II] $\lambda \lambda 6717,6731$, and the [O II] $\lambda \lambda 3727,3729$ ratios, although these lines are probably affected by the reddening corrections made to the observations.

What could enhance the $\left[\mathrm{S}_{\text {II] }} \lambda \lambda 6717,6731 / \mathrm{H} \alpha\right.$ ratio or the [O I] $\lambda 6300 / \mathrm{H} \alpha$ ratio? First, both the $\mathrm{O}$ I and the $\mathrm{S}$ II ions are very much more sensitive than the $\mathrm{N}$ in ion to the diffuse radiation field in $\mathrm{H}$ II regions. They are therefore much enhanced in the vicinity of ionization fronts, and in shadow regions behind elephant trunk features, which are obscured from direct stellar radiation and illuminated only by the diffuse nebular radiation field. Second, both are much enhanced in shock regions. Indeed, high $\left[\mathrm{S}_{\mathrm{II}}\right] / \mathrm{H} \alpha$ ratios are used as a means of identifying supernova remnants either embedded in $\mathrm{H}$ II regions or in the ISM at large, and [O I $] / \mathrm{H} \alpha$ ratios reach 0.5 or higher in shock-excited regions. Neither of these effects are taken into account in the models.

In addition, the $\mathrm{O}_{\mathrm{I}}$ ion is locked by charge-exchange reactions to the concentration of $\mathrm{H}$ I. Furthermore, the collision strength of [O I] 26300 increases with electron temperature. To produce a high $\left[\mathrm{O}_{\mathrm{I}}\right] \lambda 6300 / \mathrm{H} \alpha$ ratio in a purely photoionized region requires the presence of a hard radiation field. This could originate either as a thermal soft X-ray continuum from the shocked bubble of stellar wind material, or it could simply be an indication of a hotter subcomponent in the global stellar radiation field.

If the radiation field produced by Starburst 99 version 5 is too soft, this could be rectified in at least three ways. First, by the use of a flatter IMF in the upper mass ranges $\left(M / M_{\odot}>10\right)$. This could be possible because observationally we have very few constraints other than by use of the excitation of $\mathrm{H}$ II regions. The second is by adding another stellar component such as massexchange massive binaries to the stellar synthesis.

A third - and quite likely - possibility is that the EUV spectrum of the massive stars used in Starburst 99 version 5 may be in error. With previous versions of Starburst99 based on the CoStar models (Schaerer \& de Koter 1997), the EUV spectrum was, rather, too hard. However, Morisset et al. (2004) has demonstrated that the theoretical number of ionizing photons emitted by the central star and the shape of the ionizing spectrum is highly dependent on the atmospheric model used. At one extreme are the CoStar models, at the other extreme are found the "classical" Kurucz $(1991,1994)$ plane-parallel LTE line blanketed models used in Starburst99 version 5. The Kurucz models display the softest EUV spectra and the lowest photoionizing flux, and they are restricted to $\log g=3.0$ for the models with higher effective temperature, $T_{\text {eff }}$. In between these extremes are found three sets of models with fairly similar photoionizing flux predictions (Morisset et al. 2004), the TLUSTY models of Lanz \& Hubeny (2003) the WM-Basic models of Pauldrach et al. (2001), and the CMFGEN models of Hillier \& Miller (1998). Of these, the TLUSTY models are plane-parallel hydrostatic, while the other two are spherical dynamic atmospheres. All of this offers plenty of potential for error in the estimation of the hardness of the EUV spectrum for a given cluster mass and metallicity.

Given that the $[\mathrm{O}$ III] $\lambda 5007 / \mathrm{H} \beta$ ratio of the models also appears to be on the low side, we believe that an increase in the stellar effective temperature, either by a flattening of the IMF or by some other means offers the best chance to more properly match the theory with the observations. The models are too weak in their forbidden line strengths overall. The Stoy (1933) method (Pottasch \& Preite-Martinez 1983) of deriving the effective temperature of the exciting stars relies on measuring the total flux cooling lines (which is a measure of the mean energy per photoionization) to a recombination line (which counts the number of photoionizations). Thus, if we want to increase the absolute strengths of the forbidden lines, we have to increase the stellar effective temperature. This increase would also strongly increase the $\left[\mathrm{O}_{\mathrm{I}}\right] \lambda 6300 /$ $\mathrm{H} \alpha$ ratio by both increasing the extent of the partially ionized zone and by increasing the electron temperature within it.

A niggling uncertainty with the modeling is the mismatch between the Starburst 99 version 5 abundance set and the abundance set given in Table 1. Starburst 99 version 5 has to rely on the older stellar evolution models, which use the old value of the solar abundances. In an attempt to quantify this effect, we ran a test model with a $0.4 Z_{\odot}$ spectral synthesis cluster model from Starburst 99 embedded in a nebula with $1.0 Z_{\odot}$ (using the abundances given in Table 1). This made surprisingly little difference, changing critical line ratios by 0.1 dex or less, except for the $\left[\mathrm{O}_{\mathrm{I}}\right] \lambda 6300 / \mathrm{H} \alpha$ ratio, which increased by $34 \%$. We therefore conclude that the results presented in this paper are reasonably secure against any future adjustment of stellar atmospheric abundances.

In conclusion, the major advances in modeling made in this paper are as follows:

1. For individual $\mathrm{H}$ II regions, it becomes possible to estimate the ages of the exciting stars from the positions of the observations on $\mathrm{H}$ II region isochrones. Previously, this was not possible, since the ionization parameter and the effective temperature of the cluster (determined by the aging of its stars) produce similar effects on the emitted spectrum. The self-consistent treatment we have developed that includes the dynamical evolution of the $\mathrm{H}$ II region consistent with the properties of the stellar wind generated by the central cluster and that recognizes that the stellar wind determines the pressure in the $\mathrm{H}$ in region allows us to eliminate the use of arbitrary geometries.

2. In addition, this work gives a methodology to construct much more "realistic" integrated spectra of $\mathrm{H}$ II galaxies, something that has not been possible before in the approximations, which assume that a whole galaxy can be represented by a single spherical $\mathrm{H}$ II region with a chosen abundance and ionization parameter.

M. A. Dopita acknowledges the support of both the Australian National University and the Australian Research Council (ARC) through his ARC Australian Federation Fellowship. M. A. Dopita, R. S. Sutherland, and J. Fishera acknowledge financial support of ARC Discovery project grant DP0208445. L. J. Kewley acknowledges a Hubble Fellowship. The work by W. van Breugel was performed under the auspices of the US Department of Energy and Lawrence Livermore National Laboratory under contract W-7405Eng-48. 


\section{APPENDIX}

Here we give a summary of the strong line flux ratios with respect to $\mathrm{H} \beta$ as computed in the photoionization models. The line identifications, the ionic species, and the wavelengths of each line are given in Table 2 .

Separate tables are given for each of the abundances computed; $0.05,0.2,0.4,1.0$, and $2.0 Z_{\odot}$, and in each of these tables, line fluxes with respect to $\mathrm{H} \beta=1.0$ are given for each of the 51 lines listed in Table 2 . These spectral line intensities are given for each value of $\log \mathcal{R}$ computed; $+2,0,-2,-4$, and -6 . The quantity $R$ is that defined in the text of the paper:

$$
\mathcal{R}=\left(\frac{M_{\mathrm{cl}}}{M_{\odot}}\right)\left(\frac{P / k}{10^{4} \mathrm{~cm}^{-3} \mathrm{~K}}\right) .
$$

Each of the following five tables (Tables 3, 4, 5, 6, and 7) follows the same format.

TABLE 2

Line ID AND Wavelength List

\begin{tabular}{|c|c|c|}
\hline Line ID & Ion & $\begin{array}{c}\lambda \\
(\AA)\end{array}$ \\
\hline $01 \ldots \ldots$. & $\mathrm{H}$ I & 1215.7 \\
\hline $02 \ldots$ & $\mathrm{C}$ III] & 1909.1 \\
\hline $03 \ldots \ldots \ldots$ & $\mathrm{C}$ III] & 1911.2 \\
\hline $04 \ldots \ldots \ldots \ldots \ldots$ & {$[\mathrm{C}$ II $]$} & 2325.2 \\
\hline $05 \ldots \ldots \ldots \ldots \ldots \ldots \ldots \ldots$ & $\mathrm{Mg}$ II & 2797.9 \\
\hline 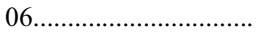 & {$[\mathrm{O}$ II $]$} & 3726.0 \\
\hline 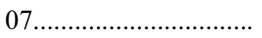 & {$[\mathrm{O}$ п] } & 3728.7 \\
\hline $08 \ldots$ & $\mathrm{H} \mathrm{I}_{\mathrm{I}}$ & 3797.9 \\
\hline ................ & $\mathrm{H}$ I & 3835.4 \\
\hline .............. & {$[\mathrm{Ne}$ III] } & 3868.7 \\
\hline 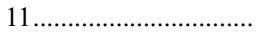 & He I & 3888.6 \\
\hline .................. & $\mathrm{H} \mathrm{I}_{\mathrm{I}}$ & 3889.1 \\
\hline $13 \ldots \ldots$ & {$[\mathrm{Ne} \mathrm{III}]$} & 3967.4 \\
\hline ............... & $\mathrm{H}$ I & 3970.1 \\
\hline $15 \ldots \ldots$. & $\mathrm{H}$ I & 4104.7 \\
\hline 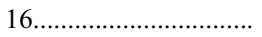 & $\mathrm{H}$ I & 4340.5 \\
\hline $17 \ldots \ldots$ & He I & 4471.5 \\
\hline $18 \ldots \ldots$ & $\mathrm{H}_{\mathrm{I}}$ & 4861.3 \\
\hline$\ldots \ldots \ldots \ldots$ & [O III] & 4958.8 \\
\hline $20 \ldots \ldots \ldots \ldots$ & [O III $]$ & 5006.8 \\
\hline $21 \ldots \ldots \ldots$ & He I & 5875.6 \\
\hline $22 \ldots \ldots$ & {$[\mathrm{O}$ I $]$} & 6300.2 \\
\hline ................ & {$[\mathrm{N}$ II $]$} & 6548.0 \\
\hline $24 \ldots \ldots \ldots \ldots \ldots$ & $\mathrm{H} \mathrm{I}$ & 6562.8 \\
\hline .................. & {$[\mathrm{N}$ II $]$} & 6583.3 \\
\hline $26 \ldots \ldots$ & He I & 6678.2 \\
\hline $27 \ldots \ldots$. & {$[\mathrm{S}$ II $]$} & 6716.3 \\
\hline $28 \ldots \ldots$. & {$[\mathrm{S}$ II $]$} & 6730.7 \\
\hline $29 \ldots \ldots$ & [Ar III] & 7135.7 \\
\hline ….................... & [Ar III] & 7751.0 \\
\hline 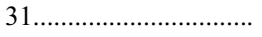 & {$[\mathrm{S}$ III $]$} & 9069.3 \\
\hline $32 \ldots \ldots \ldots \ldots \ldots \ldots \ldots \ldots$ & $\mathrm{H}$ I & 10049.4 \\
\hline $33 \ldots \ldots \ldots$ & $\mathrm{H}$ I & 10049.4 \\
\hline 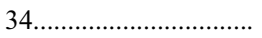 & He I & 10830.0 \\
\hline $35 \ldots \ldots$. & He I & 10833.0 \\
\hline 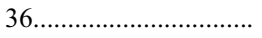 & $\mathrm{H} \mathrm{I}$ & 10938.1 \\
\hline$\ldots \ldots \ldots \ldots$ & $\mathrm{H} \mathrm{I}$ & 12818.1 \\
\hline $38 \ldots \ldots \ldots \ldots . . .$. & $\mathrm{H} \mathrm{I}$ & 18751.0 \\
\hline ................. & $\mathrm{H} \mathrm{I}$ & 26252.0 \\
\hline $40 \ldots \ldots \ldots \ldots \ldots$ & $\mathrm{H}$ I & 40512.0 \\
\hline 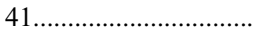 & [Ar II] & 69832.8 \\
\hline $42 \ldots \ldots \ldots \ldots \ldots \ldots \ldots \ldots$ & [Ar III] & 89892.5 \\
\hline 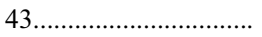 & [S IV] & 105221 \\
\hline $44 \ldots \ldots \ldots \ldots \ldots$ & {$[\mathrm{Ne}$ II $]$} & 128115 \\
\hline $45 \ldots \ldots \ldots \ldots \ldots \ldots \ldots \ldots \ldots$ & {$[\mathrm{Ne}$ III $]$} & 155513 \\
\hline 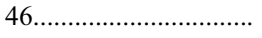 & {$[\mathrm{S}$ III $]$} & 186821 \\
\hline 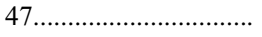 & {$[\mathrm{S}$ III $]$} & 336366 \\
\hline 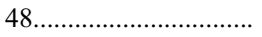 & [Si II $]$ & 347941 \\
\hline 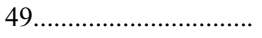 & [O III] & 517972 \\
\hline $50 \ldots \ldots \ldots \ldots \ldots \ldots$ & {$[\mathrm{N}$ III] } & 573845 \\
\hline 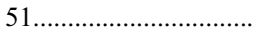 & [O III] & 883017 \\
\hline$\ldots \ldots \ldots \ldots$ & $\cdots$ & $\cdots$ \\
\hline
\end{tabular}


TABLE 3

Line Fluxes in Model H in Regions (with Respect to $\mathrm{H} \beta=1.0$ ) for the $0.05 Z_{\odot}$ Abundance $\mathrm{Set}$

\begin{tabular}{|c|c|c|c|c|c|c|c|c|c|c|c|c|c|c|c|}
\hline Line ID & $\begin{array}{c}\lambda \\
(\AA)\end{array}$ & $\begin{array}{c}F \\
(0.1 \mathrm{Myr})\end{array}$ & $\begin{array}{c}F \\
(0.5 \mathrm{Myr})\end{array}$ & $\begin{array}{c}F \\
(1.0 \mathrm{Myr})\end{array}$ & $\begin{array}{c}F \\
(1.5 \mathrm{Myr})\end{array}$ & $\begin{array}{c}F \\
(2.0 \mathrm{Myr})\end{array}$ & $\begin{array}{c}F \\
(2.5 \mathrm{Myr})\end{array}$ & $\begin{array}{c}F \\
(3.0 \mathrm{Myr})\end{array}$ & $\begin{array}{c}F \\
(3.5 \mathrm{Myr})\end{array}$ & $\begin{array}{c}F \\
(4.0 \mathrm{Myr})\end{array}$ & $\begin{array}{c}F \\
(4.5 \mathrm{Myr})\end{array}$ & $\begin{array}{c}F \\
(5.0 \mathrm{Myr})\end{array}$ & $\begin{array}{c}F \\
\text { (5.5 Myr) }\end{array}$ & $\begin{array}{c}F \\
(6.0 \mathrm{Myr})\end{array}$ & $\begin{array}{c}F \\
\text { (Mean) }\end{array}$ \\
\hline \multicolumn{16}{|c|}{$\log R=+2$} \\
\hline 8 & $1.216 \mathrm{E}+03$ & $3.584 \mathrm{E}+01$ & $3.509 \mathrm{E}+01$ & $3.477 \mathrm{E}+01$ & $3.460 \mathrm{E}+01$ & $3.439 \mathrm{E}+01$ & 191E+01 & $3.122 \mathrm{E}+01$ & $.330 \mathrm{E}+01$ & $.093 \mathrm{E}+01$ & $3.066 \mathrm{E}+01$ & $2.976 \mathrm{E}+01$ & $3.000 \mathrm{E}+01$ & 3.010 & $3.277 \mathrm{E}+01$ \\
\hline $02 .$. & $1.909 \mathrm{E}+03$ & $2.070 \mathrm{E}-01$ & $1.775 \mathrm{E}-01$ & $1.666 \mathrm{E}-01$ & $1.616 \mathrm{E}-01$ & $1.568 \mathrm{E}-01$ & $1.426 \mathrm{E}-01$ & $1.456 \mathrm{E}-01$ & $1.164 \mathrm{E}-01$ & $7.847 \mathrm{E}-02$ & $5.969 \mathrm{E}-02$ & $3.730 \mathrm{E}-02$ & $2.577 \mathrm{E}-02$ & $1.920 \mathrm{E}-02$ & $1.289 \mathrm{E}-01$ \\
\hline $03 \ldots \ldots \ldots . . .$. & $1.911 \mathrm{E}+03$ & $1.358 \mathrm{E}-01$ & $1.162 \mathrm{E}-01$ & $1.090 \mathrm{E}-01$ & $1.057 \mathrm{E}-01$ & $1.026 \mathrm{E}-01$ & $9.324 \mathrm{E}-02$ & $9.530 \mathrm{E}-02$ & $7.632 \mathrm{E}-02$ & $5.146 \mathrm{E}-02$ & $3.914 \mathrm{E}-02$ & $2.446 \mathrm{E}-02$ & $1.690 \mathrm{E}-02$ & $1.259 \mathrm{E}-02$ & $8.438 \mathrm{E}-02$ \\
\hline . & $2.325 \mathrm{E}+03$ & $6.546 \mathrm{E}-03$ & $7.732 \mathrm{E}-03$ & $8.224 \mathrm{E}-03$ & $8.454 \mathrm{E}-03$ & $8.621 \mathrm{E}-03$ & $1.037 \mathrm{E}-02$ & $1.071 \mathrm{E}-02$ & $1.156 \mathrm{E}-02$ & $1.731 \mathrm{E}-02$ & $2.024 \mathrm{E}-02$ & $2.604 \mathrm{E}-02$ & $2.708 \mathrm{E}-02$ & $2.730 \mathrm{E}-02$ & $1.235 \mathrm{E}-02$ \\
\hline & $2.798 \mathrm{E}+03$ & $1.309 \mathrm{E}-02$ & $1.552 \mathrm{E}-02$ & $1.652 \mathrm{E}-02$ & $1.701 \mathrm{E}$ & $1.736 \mathrm{E}$ & $1.801 \mathrm{E}-02$ & $1.676 \mathrm{E}$ & $1.813 \mathrm{E}$ & $2.125 \mathrm{E}$ & $2.429 \mathrm{E}$ & 2.815 & 3.24 & 3.544 & $1.934 \mathrm{E}-02$ \\
\hline & $3.726 \mathrm{E}+03$ & $4.633 \mathrm{E}-02$ & $5.721 \mathrm{E}-02$ & $6.241 \mathrm{E}$ & $6.496 \mathrm{E}$ & $6.712 \mathrm{E}$ & $8.229 \mathrm{E}-02$ & 8.516 & 9.7911 & 1.682 & 2.076 & $2.748 \mathrm{E}$ & 2.868 & 2.8601 & $1.094 \mathrm{E}-01$ \\
\hline & $3.729 \mathrm{E}+03$ & $5.745 \mathrm{E}-02$ & $8.050 \mathrm{E}-02$ & $8.978 \mathrm{E}-02$ & $9.422 \mathrm{E}-02$ & $9.790 \mathrm{E}-02$ & $1.207 \mathrm{E}-01$ & $1.229 \mathrm{E}$ & $1.316 \mathrm{~F}$ & $2.302 \mathrm{E}-01$ & $2.882 \mathrm{E}-01$ & $3.851 \mathrm{E}$ & $4.049 \mathrm{E}$ & $4.064 \mathrm{~F}$ & $1.549 \mathrm{E}-01$ \\
\hline 08. & $3.798 \mathrm{E}+03$ & $5.346 \mathrm{E}-02$ & $5.342 \mathrm{E}-02$ & $5.340 \mathrm{E}-02$ & $5.339 \mathrm{E}-02$ & $5.339 \mathrm{E}-02$ & $5.342 \mathrm{E}-02$ & $5.347 \mathrm{E}$ & $5.344 \mathrm{E}$ & $5.343 \mathrm{E}-02$ & $5.338 \mathrm{E}$ & $5.332 \mathrm{E}$ & $5.326 \mathrm{E}$ & $5.321 \mathrm{~F}$ & $5.340 \mathrm{E}-02$ \\
\hline $09 .$. & $3.835 \mathrm{E}+03$ & $7.386 \mathrm{E}-02$ & $7.379 \mathrm{E}-02$ & $7.376 \mathrm{E}-02$ & $7.375 \mathrm{E}-02$ & $7.374 \mathrm{E}-02$ & $7.378 \mathrm{E}-02$ & $7.383 \mathrm{E}-02$ & $7.378 \mathrm{E}-02$ & $7.375 \mathrm{E}-02$ & $7.369 \mathrm{E}-02$ & $7.360 \mathrm{E}-02$ & $7.352 \mathrm{E}-02$ & $7.345 \mathrm{E}-02$ & $7.374 \mathrm{E}-02$ \\
\hline $10 \ldots \ldots \ldots \ldots$ & $3.869 \mathrm{E}+03$ & $4.170 \mathrm{E}-01$ & $3.723 \mathrm{E}-01$ & $3.557 \mathrm{E}-01$ & $3.481 \mathrm{E}-01$ & $3.412 \mathrm{E}-01$ & $3.129 \mathrm{E}-01$ & $3.141 \mathrm{E}-01$ & $2.798 \mathrm{E}-01$ & $1.985 \mathrm{E}-01$ & $1.560 \mathrm{E}-01$ & $8.815 \mathrm{E}-02$ & $6.181 \mathrm{E}-02$ & $4.658 \mathrm{E}-02$ & $2.848 \mathrm{E}-01$ \\
\hline
\end{tabular}

Note.-Table 3 is available in its entirety in the electronic edition of the Astrophysical Journal Supplement. A portion is shown here for guidance regarding its form and content. 
TABLE 4

Line Fluxes in Model H il Regions (with Respect to $\mathrm{H} \beta=1.0$ ) for the $0.2 Z_{\odot}$ Abundance Set

\begin{tabular}{|c|c|c|c|c|c|c|c|c|c|c|c|c|c|c|c|}
\hline Line ID & $\begin{array}{c}\lambda \\
(\AA)\end{array}$ & $\begin{array}{c}F \\
(0.1 \mathrm{Myr})\end{array}$ & $\begin{array}{c}F \\
(0.5 \mathrm{Myr})\end{array}$ & $\begin{array}{c}F \\
(1.0 \mathrm{Myr})\end{array}$ & $\begin{array}{c}F \\
(1.5 \mathrm{Myr})\end{array}$ & $\begin{array}{c}F \\
(2.0 \mathrm{Myr})\end{array}$ & $\begin{array}{c}F \\
(2.5 \mathrm{Myr})\end{array}$ & $\begin{array}{c}F \\
(3.0 \mathrm{Myr})\end{array}$ & $\begin{array}{c}F \\
(3.5 \mathrm{Myr})\end{array}$ & $\begin{array}{c}F \\
(4.0 \mathrm{Myr})\end{array}$ & $\begin{array}{c}F \\
(4.5 \mathrm{Myr})\end{array}$ & $\begin{array}{c}F \\
(5.0 \mathrm{Myr})\end{array}$ & $\begin{array}{c}F \\
(5.5 \mathrm{Myr})\end{array}$ & $\begin{array}{c}F \\
\text { (6.0 Myr) }\end{array}$ & $\begin{array}{c}F \\
\text { (Mean) }\end{array}$ \\
\hline
\end{tabular}

$\log R=+2$

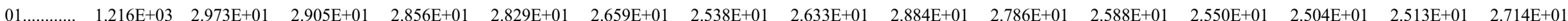

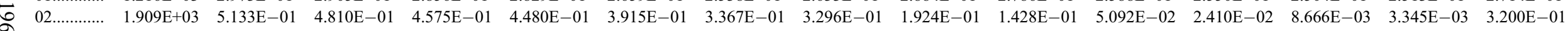

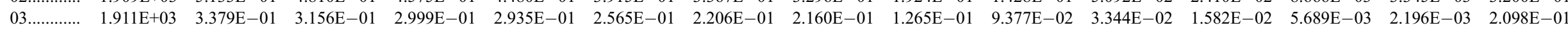

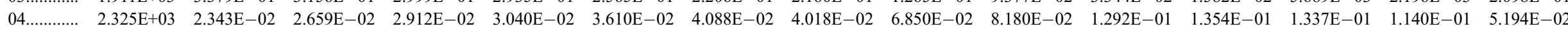

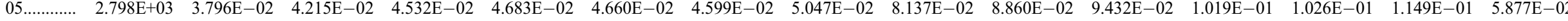

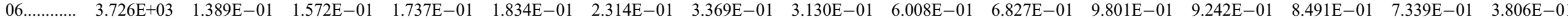

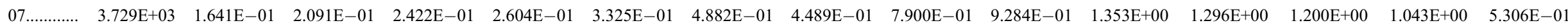

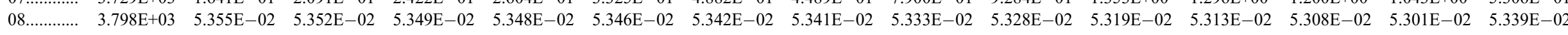

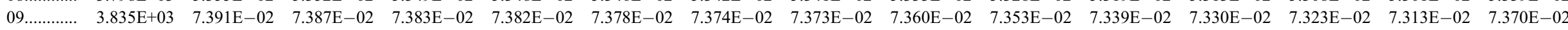

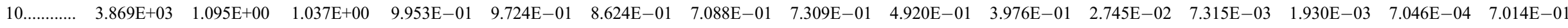

NoтE.-Table 4 is available in its entirety in the electronic edition of the Astrophysical Journal Supplement. A portion is shown here for guidance regarding its form and content. 
TABLE 5

Line Fluxes in Model H il Regions (with Respect to $\mathrm{H} \beta=1.0$ ) for the $0.4 Z_{\odot}$ Abundance Set

\begin{tabular}{|c|c|c|c|c|c|c|c|c|c|c|c|c|c|c|c|}
\hline Line ID & $\begin{array}{c}\lambda \\
(\AA)\end{array}$ & $\begin{array}{c}F \\
(0.1 \mathrm{Myr})\end{array}$ & $\begin{array}{c}F \\
(0.5 \mathrm{Myr})\end{array}$ & $\begin{array}{c}F \\
(1.0 \mathrm{Myr})\end{array}$ & $\begin{array}{c}F \\
(1.5 \mathrm{Myr})\end{array}$ & $\begin{array}{c}F \\
(2.0 \mathrm{Myr})\end{array}$ & $\begin{array}{c}F \\
(2.5 \mathrm{Myr})\end{array}$ & $\begin{array}{c}F \\
(3.0 \mathrm{Myr})\end{array}$ & $\begin{array}{c}F \\
(3.5 \mathrm{Myr})\end{array}$ & $\begin{array}{c}F \\
(4.0 \mathrm{Myr})\end{array}$ & $\begin{array}{c}F \\
(4.5 \mathrm{Myr})\end{array}$ & $\begin{array}{c}F \\
(5.0 \mathrm{Myr})\end{array}$ & $\begin{array}{c}F \\
(5.5 \mathrm{Myr})\end{array}$ & $\begin{array}{c}F \\
(6.0 \mathrm{Myr})\end{array}$ & $\begin{array}{c}F \\
\text { (Mean) }\end{array}$ \\
\hline \multicolumn{16}{|c|}{$\log R=+2$} \\
\hline & $16 \mathrm{E}+03$ & $701 \mathrm{E}+01$ & $611 \mathrm{E}+01$ & $544 \mathrm{E}+01$ & $456 \mathrm{E}+01$ & $408 \mathrm{E}-$ & $37 \mathrm{E}+01$ & $59 \mathrm{E}$ & $596 \mathrm{E}$ & $28 \mathrm{~F}$ & $427 \mathrm{E}$ & 01 & 01 & .373 & +01 \\
\hline & $1.909 \mathrm{E}+03$ & $120 \mathrm{E}-01$ & $4.630 \mathrm{E}-01$ & $4.240 \mathrm{E}-01$ & $390 \mathrm{E}-01$ & $200 \mathrm{E}-01$ & $120 \mathrm{E}-01$ & $1.800 \mathrm{E}-01$ & $1.430 \mathrm{E}-01$ & $1.100 \mathrm{E}-01$ & $2.020 \mathrm{E}-02$ & $2.870 \mathrm{E}-02$ & $1.700 \mathrm{E}-02$ & $3.840 \mathrm{E}-04$ & $2.510 \mathrm{E}-01$ \\
\hline $03 \ldots \ldots \ldots . .$. & $1.911 \mathrm{E}+03$ & $.390 \mathrm{E}-01$ & $3.050 \mathrm{E}-01$ & $2.780 \mathrm{E}-01$ & $2.220 \mathrm{E}-01$ & $100 \mathrm{E}-01$ & $390 \mathrm{E}-01$ & $1.180 \mathrm{E}$ & $9.400 \mathrm{E}-02$ & $7.250 \mathrm{E}-02$ & $1.330 \mathrm{E}-02$ & $1.890 \mathrm{E}-02$ & 1.110 & 2.530 & $1.650 \mathrm{E}-01$ \\
\hline $04 \ldots \ldots \ldots \ldots$ & $2.325 \mathrm{E}+03$ & $4.160 \mathrm{E}-02$ & $4.600 \mathrm{E}-02$ & $5.010 \mathrm{E}-02$ & $5.400 \mathrm{E}-02$ & $5.830 \mathrm{E}-02$ & $6.860 \mathrm{E}-02$ & $7.980 \mathrm{E}$ & $1.550 \mathrm{E}-01$ & $1.730 \mathrm{E}-01$ & $1.890 \mathrm{E}-01$ & $1.890 \mathrm{E}-01$ & $1.800 \mathrm{E}-01$ & $1.190 \mathrm{E}-01$ & $8.800 \mathrm{E}-02$ \\
\hline $05 .$. & $2.798 \mathrm{E}+03$ & $5.180 \mathrm{E}-02$ & $5.610 \mathrm{E}-02$ & $5.950 \mathrm{E}-02$ & $5.750 \mathrm{E}-02$ & $5.770 \mathrm{E}-02$ & $5.240 \mathrm{E}-02$ & $6.220 \mathrm{E}-02$ & $1.410 \mathrm{E}-01$ & $1.440 \mathrm{E}-01$ & $1.420 \mathrm{E}-01$ & $1.690 \mathrm{E}-01$ & $1.740 \mathrm{E}-01$ & $1.440 \mathrm{E}-01$ & $8.000 \mathrm{E}-02$ \\
\hline $06 \ldots \ldots \ldots \ldots$ & $3.726 \mathrm{E}+03$ & $2.130 \mathrm{E}-01$ & $2.340 \mathrm{E}-01$ & $2.570 \mathrm{E}-01$ & $3.050 \mathrm{E}-01$ & $4.240 \mathrm{E}-01$ & $5.380 \mathrm{E}-01$ & $6.300 \mathrm{E}-01$ & $1.160 \mathrm{E}+00$ & $1.260 \mathrm{E}+00$ & $1.120 \mathrm{E}+00$ & $1.130 \mathrm{E}+00$ & $1.060 \mathrm{E}+00$ & $7.330 \mathrm{E}-01$ & $6.000 \mathrm{E}-01$ \\
\hline $07 \ldots \ldots \ldots . . .$. & $3.729 \mathrm{E}+03$ & $2.270 \mathrm{E}-01$ & $2.950 \mathrm{E}-01$ & $3.480 \mathrm{E}-01$ & $4.230 \mathrm{E}-01$ & $5.990 \mathrm{E}-01$ & $7.660 \mathrm{E}-01$ & $8.910 \mathrm{E}-01$ & $1.500 \mathrm{E}+00$ & $1.690 \mathrm{E}+00$ & $1.530 \mathrm{E}+00$ & $1.570 \mathrm{E}+00$ & $1.490 \mathrm{E}+00$ & $1.040 \mathrm{E}+00$ & $8.230 \mathrm{E}-01$ \\
\hline & $3.798 \mathrm{E}+03$ & $5.340 \mathrm{E}-02$ & $5.330 \mathrm{E}-02$ & $5.330 \mathrm{E}-02$ & $5.320 \mathrm{E}-02$ & $5.320 \mathrm{E}-02$ & $5.310 \mathrm{E}-02$ & $5.310 \mathrm{E}-02$ & $5.310 \mathrm{E}-02$ & $5.310 \mathrm{E}-02$ & $5.290 \mathrm{E}-02$ & $5.300 \mathrm{E}-02$ & $5.290 \mathrm{E}-02$ & $5.280 \mathrm{E}-02$ & $5.300 \mathrm{E}-02$ \\
\hline & $3.835 \mathrm{E}+03$ & $7.370 \mathrm{E}-02$ & $7.360 \mathrm{E}-02$ & $7.350 \mathrm{E}-02$ & $7.340 \mathrm{E}-02$ & $7.340 \mathrm{E}-02$ & $7.330 \mathrm{E}-02$ & $7.320 \mathrm{E}-02$ & $7.330 \mathrm{E}-02$ & $7.320 \mathrm{E}-02$ & $7.300 \mathrm{E}-02$ & $7.300 \mathrm{E}-02$ & $7.300 \mathrm{E}-02$ & $7.270 \mathrm{E}-02$ & $7.300 \mathrm{E}-02$ \\
\hline $10 \ldots \ldots \ldots$ & $3.869 \mathrm{E}+03$ & $1.280 \mathrm{E}+00$ & $1.190 \mathrm{E}+00$ & $1.120 \mathrm{E}+00$ & $9.550 \mathrm{E}-01$ & $8.180 \mathrm{E}-01$ & $5.310 \mathrm{E}-01$ & $4.120 \mathrm{E}-01$ & $3.350 \mathrm{E}-01$ & $1.550 \mathrm{E}-01$ & $3.900 \mathrm{E}-02$ & $9.110 \mathrm{E}-02$ & $1.790 \mathrm{E}-02$ & $2.230 \mathrm{E}-05$ & $6.390 \mathrm{E}-01$ \\
\hline
\end{tabular}

Note.-Table 5 is available in its entirety in the electronic edition of the Astrophysical Journal Supplement. A portion is shown here for guidance regarding its form and content. 
TABLE 6

Line Fluxes in Model H il Regions (with Respect to $\mathrm{H} \beta=1.0$ ) for the $1.0 Z_{\odot}$ Abundance Set

\begin{tabular}{|c|c|c|c|c|c|c|c|c|c|c|c|c|c|c|c|}
\hline Line ID & $\begin{array}{c}\lambda \\
(\AA)\end{array}$ & $\begin{array}{c}F \\
(0.1 \mathrm{Myr})\end{array}$ & $\begin{array}{c}F \\
(0.5 \mathrm{Myr})\end{array}$ & $\begin{array}{c}F \\
(1.0 \mathrm{Myr})\end{array}$ & $\begin{array}{c}F \\
(1.5 \mathrm{Myr})\end{array}$ & $\begin{array}{c}F \\
(2.0 \mathrm{Myr})\end{array}$ & $\begin{array}{c}F \\
(2.5 \mathrm{Myr})\end{array}$ & $\begin{array}{c}F \\
(3.0 \mathrm{Myr})\end{array}$ & $\begin{array}{c}F \\
(3.5 \mathrm{Myr})\end{array}$ & $\begin{array}{c}F \\
(4.0 \mathrm{Myr})\end{array}$ & $\begin{array}{c}F \\
(4.5 \mathrm{Myr})\end{array}$ & $\begin{array}{c}F \\
(5.0 \mathrm{Myr})\end{array}$ & $\begin{array}{c}F \\
(5.5 \mathrm{Myr})\end{array}$ & $\begin{array}{c}F \\
(6.0 \mathrm{Myr})\end{array}$ & $\begin{array}{c}F \\
\text { (Mean) }\end{array}$ \\
\hline \multicolumn{16}{|c|}{$\log R=+2$} \\
\hline 0 & $1.216 \mathrm{E}+03$ & $2.608 \mathrm{E}+01$ & $2.515 \mathrm{E}+01$ & $2.445 \mathrm{E}+01$ & $2.400 \mathrm{E}+01$ & $365 \mathrm{E}+01$ & $2.379 \mathrm{E}+01$ & $2.365 \mathrm{E}+01$ & $2.398 \mathrm{E}+01$ & $2.412 \mathrm{E}+01$ & $2.412 \mathrm{E}+01$ & $2.379 \mathrm{E}+01$ & $2.368 \mathrm{E}+01$ & $2.341 \mathrm{E}+01$ & $2.406 \mathrm{E}+01$ \\
\hline $02 .$. & $1.909 \mathrm{E}+03$ & $1.565 \mathrm{E}-01$ & $1.114 \mathrm{E}-01$ & $7.189 \mathrm{E}-02$ & $6.311 \mathrm{E}-02$ & $3.646 \mathrm{E}-02$ & $1.682 \mathrm{E}-02$ & $4.366 \mathrm{E}-02$ & $2.119 \mathrm{E}-02$ & $2.199 \mathrm{E}-02$ & $2.151 \mathrm{E}-02$ & $1.118 \mathrm{E}-03$ & $1.538 \mathrm{E}-05$ & $1.127 \mathrm{E}-05$ & $4.758 \mathrm{E}-02$ \\
\hline 03. & $1.911 \mathrm{E}+03$ & $1.053 \mathrm{E}-01$ & $7.403 \mathrm{E}-02$ & $4.756 \mathrm{E}-02$ & $4.167 \mathrm{E}-02$ & $2.406 \mathrm{E}-02$ & $1.111 \mathrm{E}-02$ & $2.879 \mathrm{E}-02$ & $1.400 \mathrm{E}-02$ & $1.452 \mathrm{E}-02$ & $1.419 \mathrm{E}-02$ & $7.383 \mathrm{E}-04$ & $1.017 \mathrm{E}-05$ & $7.442 \mathrm{E}-06$ & $3.150 \mathrm{E}-02$ \\
\hline $04 .$. & $2.325 \mathrm{E}+03$ & $6.242 \mathrm{E}-02$ & $6.247 \mathrm{E}-02$ & $6.585 \mathrm{E}-02$ & $7.803 \mathrm{E}-02$ & $8.979 \mathrm{E}-02$ & $1.058 \mathrm{E}-01$ & $9.729 \mathrm{E}-02$ & $1.485 \mathrm{E}-01$ & $1.864 \mathrm{E}-01$ & $2.170 \mathrm{E}-01$ & $9.894 \mathrm{E}-02$ & $4.642 \mathrm{E}-02$ & $4.425 \mathrm{E}-02$ & $9.857 \mathrm{E}-02$ \\
\hline $05 \ldots \ldots \ldots \ldots$ & $2.798 \mathrm{E}+03$ & $4.392 \mathrm{E}-02$ & $4.344 \mathrm{E}-02$ & $3.896 \mathrm{E}-02$ & $3.744 \mathrm{E}-02$ & $3.414 \mathrm{E}-02$ & $3.704 \mathrm{E}-02$ & $6.955 \mathrm{E}-02$ & $9.130 \mathrm{E}-02$ & $1.318 \mathrm{E}-01$ & $1.683 \mathrm{E}-01$ & $8.769 \mathrm{E}-02$ & $5.164 \mathrm{E}-02$ & $5.138 \mathrm{E}-02$ & $5.584 \mathrm{E}-02$ \\
\hline 06. & $3.726 \mathrm{E}+03$ & $3.452 \mathrm{E}-01$ & $3.606 \mathrm{E}-01$ & $4.520 \mathrm{E}-01$ & $6.413 \mathrm{E}-01$ & $7.169 \mathrm{E}-01$ & $7.933 \mathrm{E}-01$ & $6.041 \mathrm{E}-01$ & $9.404 \mathrm{E}-01$ & $1.061 \mathrm{E}+00$ & $1.127 \mathrm{E}+00$ & $6.203 \mathrm{E}-01$ & $3.598 \mathrm{E}-01$ & $3.362 \mathrm{E}-01$ & $6.755 \mathrm{E}-01$ \\
\hline 07................. & $3.729 \mathrm{E}+03$ & $3.036 \mathrm{E}-01$ & $4.006 \mathrm{E}-01$ & $5.619 \mathrm{E}-01$ & $8.402 \mathrm{E}-01$ & $9.726 \mathrm{E}-01$ & $1.064 \mathrm{E}+00$ & $8.306 \mathrm{E}-01$ & $1.241 \mathrm{E}+00$ & $1.395 \mathrm{E}+00$ & $1.505 \mathrm{E}+00$ & $8.357 \mathrm{E}-01$ & $4.905 \mathrm{E}-01$ & $4.682 \mathrm{E}-01$ & $8.885 \mathrm{E}-01$ \\
\hline 08. & $3.798 \mathrm{E}+03$ & $5.279 \mathrm{E}-02$ & $5.271 \mathrm{E}-02$ & $5.263 \mathrm{E}-02$ & $5.262 \mathrm{E}-02$ & $5.257 \mathrm{E}-02$ & $5.253 \mathrm{E}-02$ & $5.257 \mathrm{E}-02$ & $5.258 \mathrm{E}-02$ & $5.262 \mathrm{E}-02$ & $5.263 \mathrm{E}-02$ & $5.239 \mathrm{E}-02$ & $5.215 \mathrm{E}-02$ & $5.211 \mathrm{E}-02$ & $5.259 \mathrm{E}-02$ \\
\hline $09 \ldots \ldots \ldots \ldots$ & $3.835 \mathrm{E}+03$ & $7.278 \mathrm{E}-02$ & $7.265 \mathrm{E}-02$ & $7.252 \mathrm{E}-02$ & $7.250 \mathrm{E}-02$ & $7.241 \mathrm{E}-02$ & $7.235 \mathrm{E}-02$ & $7.241 \mathrm{E}-02$ & $7.244 \mathrm{E}-02$ & $7.249 \mathrm{E}-02$ & $7.251 \mathrm{E}-02$ & $7.215 \mathrm{E}-02$ & $7.181 \mathrm{E}-02$ & $7.175 \mathrm{E}-02$ & $7.245 \mathrm{E}-02$ \\
\hline $10 \ldots \ldots \ldots$ & $3.869 \mathrm{E}+03$ & $6.963 \mathrm{E}-01$ & $5.581 \mathrm{E}-01$ & $3.546 \mathrm{E}-01$ & $2.094 \mathrm{E}-01$ & $7.073 \mathrm{E}-02$ & $1.183 \mathrm{E}-02$ & $2.230 \mathrm{E}-01$ & $2.263 \mathrm{E}-02$ & $4.670 \mathrm{E}-02$ & $6.713 \mathrm{E}-02$ & $4.246 \mathrm{E}-04$ & $3.039 \mathrm{E}-06$ & $3.187 \mathrm{E}-06$ & $1.798 \mathrm{E}-01$ \\
\hline
\end{tabular}

Nоте.-Table 6 is available in its entirety in the electronic edition of the Astrophysical Journal Supplement. A portion is shown here for guidance regarding its form and content. 
TABLE 7

Line Fluxes in Model H il Regions (with Respect to $\mathrm{H} \beta=1.0$ ) for the $2.0 Z_{\odot}$ Abundance Set

\begin{tabular}{|c|c|c|c|c|c|c|c|c|c|c|c|c|c|c|c|}
\hline Line ID & $\begin{array}{c}\lambda \\
(\AA)\end{array}$ & $\begin{array}{c}F \\
(0.1 \mathrm{Myr})\end{array}$ & $\begin{array}{c}F \\
(0.5 \mathrm{Myr})\end{array}$ & $\begin{array}{c}F \\
(1.0 \mathrm{Myr})\end{array}$ & $\begin{array}{c}F \\
(1.5 \mathrm{Myr})\end{array}$ & $\begin{array}{c}F \\
(2.0 \mathrm{Myr})\end{array}$ & $\begin{array}{c}F \\
(2.5 \mathrm{Myr})\end{array}$ & $\begin{array}{c}F \\
(3.0 \mathrm{Myr})\end{array}$ & $\begin{array}{c}F \\
(3.5 \mathrm{Myr})\end{array}$ & $\begin{array}{c}F \\
(4.0 \mathrm{Myr})\end{array}$ & $\begin{array}{c}F \\
(4.5 \mathrm{Myr})\end{array}$ & $\begin{array}{c}F \\
(5.0 \mathrm{Myr})\end{array}$ & $\begin{array}{c}F \\
(5.5 \mathrm{Myr})\end{array}$ & $\begin{array}{c}F \\
(6.0 \mathrm{Myr})\end{array}$ & $\begin{array}{c}F \\
\text { (Mean) }\end{array}$ \\
\hline \multicolumn{16}{|c|}{$\log R=+2$} \\
\hline $01 \ldots$ & $1.216 \mathrm{E}+03$ & $2.697 \mathrm{E}+01$ & $2.589 \mathrm{E}+01$ & $2.516 \mathrm{E}+01$ & $2.456 \mathrm{E}+01$ & $2.445 \mathrm{E}+01$ & $.393 \mathrm{E}+01$ & $2.495 \mathrm{E}+01$ & $2.451 \mathrm{E}+01$ & $2.523 \mathrm{E}+01$ & $2.465 \mathrm{E}+01$ & $2.439 \mathrm{E}+01$ & $2.405 \mathrm{E}+01$ & $2.385 \mathrm{E}+01$ & $2.480 \mathrm{E}+01$ \\
\hline $02 .$. & $1.909 \mathrm{E}+03$ & $1.614 \mathrm{E}-02$ & $8.837 \mathrm{E}-03$ & $7.901 \mathrm{E}-03$ & $2.262 \mathrm{E}-03$ & $4.168 \mathrm{E}-04$ & $.548 \mathrm{E}-05$ & $3.528 \mathrm{E}-05$ & $1.906 \mathrm{E}-03$ & $1.211 \mathrm{E}-03$ & $2.842 \mathrm{E}-04$ & $8.333 \mathrm{E}-04$ & $2.103 E-03$ & $2.342 \mathrm{E}-03$ & $3.288 \mathrm{E}-03$ \\
\hline 03. & $1.911 \mathrm{E}+03$ & $1.113 \mathrm{E}-02$ & $5.952 \mathrm{E}-03$ & $5.270 \mathrm{E}-03$ & $1.503 \mathrm{E}-03$ & $2.769 \mathrm{E}-04$ & $1.028 \mathrm{E}-05$ & $2.353 \mathrm{E}-05$ & $1.266 \mathrm{E}-03$ & $8.084 \mathrm{E}-04$ & $1.889 \mathrm{E}-04$ & $5.530 \mathrm{E}-04$ & $1.393 \mathrm{E}-03$ & $1.550 \mathrm{E}-03$ & $2.202 \mathrm{E}-03$ \\
\hline $04 \ldots \ldots \ldots . . .$. & $2.325 \mathrm{E}+03$ & $4.521 \mathrm{E}-02$ & $4.181 \mathrm{E}-02$ & $105 \mathrm{E}-02$ & $3.081 \mathrm{E}-02$ & $2.057 \mathrm{E}-02$ & $5.543 \mathrm{E}-03$ & $5.713 \mathrm{E}-03$ & $5.553 \mathrm{E}-02$ & $7.895 \mathrm{E}-02$ & $3.801 \mathrm{E}-02$ & $6.657 \mathrm{E}-02$ & $1.042 \mathrm{E}-01$ & $1.096 \mathrm{E}-01$ & $3.530 \mathrm{E}-02$ \\
\hline $05 \ldots \ldots \ldots \ldots . .$. & $2.798 \mathrm{E}+03$ & $2.078 \mathrm{E}-02$ & $1.770 \mathrm{E}-02$ & $1.553 \mathrm{E}-02$ & $1.221 \mathrm{E}-02$ & $9.749 \mathrm{E}-03$ & $3.898 \mathrm{E}-03$ & $2.839 \mathrm{E}-03$ & $4.407 \mathrm{E}-02$ & $6.292 \mathrm{E}-02$ & $3.510 \mathrm{E}-02$ & $5.739 \mathrm{E}-02$ & $8.525 \mathrm{E}-02$ & $8.970 \mathrm{E}-02$ & $2.007 \mathrm{E}-02$ \\
\hline $06 \ldots \ldots \ldots$ & $3.726 \mathrm{E}+03$ & $3.658 \mathrm{E}-01$ & $3.963 \mathrm{E}-01$ & $4.300 \mathrm{E}-01$ & $3.207 \mathrm{E}-01$ & $2.239 \mathrm{E}-01$ & $9.018 \mathrm{E}-02$ & $9.889 \mathrm{E}-02$ & $4.043 \mathrm{E}-01$ & $4.867 \mathrm{E}-01$ & $2.878 \mathrm{E}-01$ & $4.011 \mathrm{E}-01$ & $5.265 \mathrm{E}-01$ & $5.346 \mathrm{E}-01$ & $3.145 \mathrm{E}-01$ \\
\hline 07.............. & $3.729 \mathrm{E}+03$ & $2.628 \mathrm{E}-01$ & $3.728 \mathrm{E}-01$ & $4.782 \mathrm{E}-01$ & $3.989 \mathrm{E}-01$ & $2.844 \mathrm{E}-01$ & $1.222 \mathrm{E}-01$ & $1.174 \mathrm{E}-01$ & $5.056 \mathrm{E}-01$ & $5.337 \mathrm{E}-01$ & $3.539 \mathrm{E}-01$ & $5.090 \mathrm{E}-01$ & $6.943 \mathrm{E}-01$ & $7.199 \mathrm{E}-01$ & $3.656 \mathrm{E}-01$ \\
\hline $08 \ldots \ldots \ldots \ldots$ & $3.798 \mathrm{E}+03$ & $5.220 \mathrm{E}-02$ & $5.209 \mathrm{E}-02$ & $5.206 \mathrm{E}-02$ & $5.190 \mathrm{E}-02$ & $5.176 \mathrm{E}-02$ & $5.143 \mathrm{E}-02$ & $5.152 \mathrm{E}-02$ & $5.196 \mathrm{E}-02$ & $5.202 \mathrm{E}-02$ & $5.181 \mathrm{E}-02$ & $5.193 \mathrm{E}-02$ & $5.203 \mathrm{E}-02$ & $5.201 \mathrm{E}-02$ & $5.187 \mathrm{E}-02$ \\
\hline $09 \ldots \ldots \ldots . .$. & $3.835 \mathrm{E}+03$ & $7.189 \mathrm{E}-02$ & $7.173 \mathrm{E}-02$ & $7.169 \mathrm{E}-02$ & $7.146 \mathrm{E}-02$ & $7.125 \mathrm{E}-02$ & $7.080 \mathrm{E}-02$ & $7.091 \mathrm{E}-02$ & $7.154 \mathrm{E}-02$ & $7.164 \mathrm{E}-02$ & $7.133 \mathrm{E}-02$ & $7.149 \mathrm{E}-02$ & $7.164 \mathrm{E}-02$ & $7.161 \mathrm{E}-02$ & $7.141 \mathrm{E}-02$ \\
\hline $10 \ldots \ldots \ldots \ldots$ & $3.869 \mathrm{E}+03$ & $1.436 \mathrm{E}-01$ & $6.575 \mathrm{E}-02$ & $2.366 \mathrm{E}-02$ & $2.240 \mathrm{E}-03$ & $1.568 \mathrm{E}-04$ & $4.307 \mathrm{E}-06$ & $7.026 \mathrm{E}-06$ & $5.930 \mathrm{E}-03$ & $1.417 \mathrm{E}-03$ & $1.949 \mathrm{E}-04$ & $1.511 \mathrm{E}-03$ & $4.387 \mathrm{E}-03$ & $4.275 \mathrm{E}-03$ & $1.421 \mathrm{E}-02$ \\
\hline
\end{tabular}

Nоте.-Table 7 is available in its entirety in the electronic edition of the Astrophysical Journal Supplement. A portion is shown here for guidance regarding its form and content. 
Alloin, D., et al. 1979, A\&A, 78, 200

Anders, E., \& Grevesse, N. 1989, Geochim. Cosmochim. Acta, 53, 197

Asplund, M., Grevesse, N., \& Sauval, A. J. 2005, in ASP Conf. Ser. 336, Cosmic Abundances as Records of Stellar Evolution, ed. T. G. Barnes \& F. N. Bash (San Francisco: ASP), 25

Baldwin, J. A., Phillips, M. M., \& Terlevich, R. 1981, PASP, 93, 5 (BPT)

Bresolin, F., Garnett, D. R., \& Kennicutt, R. C., Jr. 2004, ApJ, 615, 228

Castor, J., McCray, R., \& Weaver, R. 1975, ApJ, 200, L107

Cardelli, J. A., Clayton, G. C., \& Mathis, J. S. 1989, ApJ, 345, 245

Dennefeld, M., \& Stasinska, G. 1983, A\&A, 118, 234

Dopita, M. A., \& Evans, I. N. 1986, ApJ, 307, 431

Dopita, M. A., Kewley, L. J., Heisler, C. A., \& Sutherland, R. S. 2000, ApJ, 542,224

Dopita, M. A., Groves, B. A., Sutherland, R. S., Binette, L., \& Cecil, G. 2002, ApJ, 572, 753

Dopita, M. A., et al. 2005, ApJ, 619, 755 (Paper I)

2006a, ApJ, 639, 788

2006b, ApJ, 647, 244 (Paper II)

Edmunds, M. G., \& Pagel, B. E., J. 1984, MNRAS, 211, 507

Fischera, J., Dopita, M. A, \& Sutherland, R. S. 2003, ApJ, 599, L21

Fischera, J., \& Dopita, M. A 2005, ApJ, 619, 340

Garnett, D. R., et al. 1999, ApJ, 513, 168

Giveon, U., Sternberg, A., Lutz, D., Feuchtgruber, H., \& Pauldrach, A. W., A. 2002, ApJ, 566, 880

Groves, B. A., Dopita, M. A., \& Sutherland, R. S. 2004, ApJS, 153, 9

Hoopes, C. G., Waterbos, R. A. M., \& Greenwalt, B. E. 1996, AJ, 112, 1429

Houck, J. R., et al. 2004, ApJS, 154, 18

Hillier, D. J., \& Miller, D. L. 1998, ApJ, 496, 407

Jansen, R. A., Franx, M., Fabricant, D., \& Caldwell, N. 2000a, ApJS, 126, 271

Kennicutt, R. C., Jr., \& Garnett, D. R. 1996, ApJ, 456, 504

Kewley, L. J., Heisler, C. A., Dopita, M. A., \& Lumsden, S. 2001a, ApJS, 132, 37

Kewley, L. J., Geller, M. J., Jansen, R. A., \& Dopita, M. 2002, AJ, 124, 3135

Kewley, L., \& Kobulnicky, H. A. 2005, in ASSL Conf. Ser. 329, StarburstsFrom 30 Doradus to Lyman Break Galaxies, ed. R. de Grijs \& R. M. González Delgado (Berlin: Springer), 303

Kimura, H., Mann, I., \& Jessberger, E. K. 2003, ApJ, 582, 846

Kobulnicky, H. A., Kennicutt, R. C., \& Pizagno, J. L. 1999, ApJ, 514, 544

Kobulnicky, H. A., \& Zaritsky, D. 1999, ApJ, 511, 118

Kurucz, R. L. 1991, in Stellar Atmospheres: Beyond Classical Models, ed. L. Crivellari, I. Hubeny, \& D. Hummer (Dordrecht: Reidel), 441 . 1992, in IAU Symp. 149, The Stellar Populations of Galaxies, ed. B. Barbuy \& A. Renzini (Dordrecht: Kluwer), 225

- 1994, Kurucz CD-ROM no. 19, Solar Abundance Model Atmospheres for $0,1,2,4$ and $8 \mathrm{~km} / \mathrm{s}$ (Cambridge: $\mathrm{SAO}$ )

Lanz, T., \& Hubeny, I. 2003, ApJS, 146, 417 (erratum 147, 225)

Lejeune, Th., Cuisinier, F., \& Buser, R. 1997, A\&AS, 125, 229
REFERENCES

Leitherer, C., Gruenwald, R., \& Schmutz, W. 1992, in Physics of Nearby Galaxies, ed. T. X. Thuan, C. Balkowski, \& J. T. T. Van (Gif-sur-Yvette: Éditions Frontiéres), 257

Leitherer, C., Schaerer, D., Goldader, J. D., González Delgado, R. M., R. C., Kune, D. F., de Mello, D., F., Devost, D., \& Heckman, T. M. 1999, ApJS, 123,3

Luridiana \& Peimbert, M. 2001, ApJ, 553, 633

Martin, C. L. 1997, ApJ, 491, 561

McCall, M. L., Rybski, P. M., \& Shields, G. A. 1985, ApJS, 57, 1

McGaugh, S. S. 1991, ApJ, 380, 140

Miller, G. E., \& Scalo, J. M. 1979, ApJS, 41, 513

Morisset, C., Schaerer, D., Bouret, J.-C., \& Martins, F. 2004, A\&A, 415, 577

Moustakas, J., \& Kennicutt, R. C., Jr. 2005, preprint (asto-ph/0511731) . 2006, ApJS, in press

Oey, M. S., Shields, J. C., Dopita, M. A., \& Smith, R. C. 2002, Rev. Mex. AA, 12,77

Oey, M. S., et al. 2006, AJ, in press

Pagel, B. E. J., Edmunds, M. G., \& Smith, G. 1980, MNRAS, 193, 219

Pagel, B. E. J., Simonson, E. A., Terlevich, R. J., \& Edmunds, M. G. 1992, MNRAS, 255, 325

Pagel, B. E. J., et al 1979, MNRAS, 189, 95

Pauldrach, A. W. A., Hoffman, T. L., \& Lennon, M. 2001, A\&A, 375, 161

Peeters, E., et al. 2002, A\&A, 381, 571

Pettini, M., \& Pagel, B. E. J. 2004, MNRAS, 348, L59

Pilyugin, L. S. 2000, A\&A, 362, 325

Pottasch, A. S., \& Preite-Martinez, A. 1983, A\&A, 126, 31

Reynolds, R. J. 2004, Adv. Space Res., 34, 27

Roy, J.-R., \& Walsh, J. R. 1997, MNRAS, 288, 715

Russell, S. C., \& Dopita, M. A. 1992, ApJ, 384, 508

Schaerer, D., \& de Koter, A. 1997, A\&A, 322, 598

Skillman, E. D., Kennicutt, R. C., \& Hodge, P. W. 1989, ApJ, 347, 875

Smith, L. J., Norris, R. P. F., \& Crowther, P. A. 2002, MNRAS, 337, 1309

Stasinska, G., \& Izotov, Y. 2003, A\&A, 397, 71

Steidel, C C., Giavalisco, M., Pettini, M., Dickinson, M., \& Adelberger, K. L. 1996, ApJ, 462, L17

Stoy, R. H. 1933, MNRAS, 93, 588

Sutherland, R. S., \& Dopita, M. A. 1993, ApJS, 88, 253

Torres-Peimbert, S., Peimbert, M., \& Fierro, J. 1989, ApJ, 345, 186

van Zee, L. et al. 1998, AJ, 116, 2805

Veilleux, S., \& Osterbrock, D. E. 1987, ApJS, 63, 295 (V\&O)

Verma, A., Lutz, D., Sturm, E., Sternberg, A., Genzel, R., \& Vacca, W. 2003, A\&A, 403, 829

Walsh, J. R., \& Roy, J.-R. 1997, MNRAS, 288, 726

Wang, J., Heckman, T. M., \& Lehnert, M. D. 1998, ApJ, 509, 93 1999, ApJ, 515, 97

Zaritsky, D., Kennicutt, R. C., \& Huchra, J. P. 1994, ApJ, 420, 87 